\title{
Global Stabilization of Nonlinear Systems Based on Vector Control Lyapunov Functions
}

\author{
Iasson Karafyllis and Zhong-Ping Jiang, Fellow, IEEE
}

\begin{abstract}
This paper studies the use of vector Lyapunov functions for the design of globally stabilizing feedback laws for nonlinear systems. Recent results on vector Lyapunov functions are utilized. The main result of the paper shows that the existence of a vector control Lyapunov function is a necessary and sufficient condition for the existence of a smooth globally stabilizing feedback. Applications to nonlinear systems are provided: practically checkable sufficient conditions are proposed to guarantee the existence of a smooth globally stabilizing feedback law. The obtained results are applied to the problem of the stabilization of an equilibrium point of a reaction network taking place in a continuous stirred tank reactor.
\end{abstract}

Index Terms-Feedback stabilization, Lyapunov functions, nonlinear systems.

\section{INTRODUCTION}

$\mathbf{V}$ ECTOR Lyapunov functions have been used for a long time in stability theory for nonlinear systems (see [3], [7], [8], [13]-[16], [18], [19], [23]-[26], [32] and the references therein). Vector Lyapunov functions were first introduced by Bellman in [3] and have been acknowledged to be a more flexible tool for proving stability than the usual single Lyapunov function. In view of emerging control applications related to complex large-scale dynamic systems, recently we have initiated an alternative approach to stability theory based on vector Lyapunov functions. More specifically, we have established vector Lyapunov theorems in [13]-[16] by means of small-gain analysis, as compared with the differential inequality approach proposed in classical vector Lyapunov results as in [3], [8], [18], [19], [23]. A fundamentally novel feature of the small-gain approach is that the differential inequalities in question are no longer required to hold simultaneously at every point of the state space.

The use of vector Lyapunov functions in control theory is not frequent. Exceptions are the works [24], [25], [26]. However, it seems reasonable to think that the flexibility shown by vector Lyapunov functions in stability theory can be utilized to our advantage for feedback control design in complex systems. The purpose of the present work is to show that this is indeed the case.

The main result of the present work (Theorem 3.4) is a direct extension of the well-known Artstein-Sontag theorem (see [2], [6], [29], [30]) to the case of vector Lyapunov functions. Therefore, the term Vector Control Lyapunov Function (VCLF) is appropriate. The term "Vector Control Lyapunov Function" was

\footnotetext{
Manuscript received January 19, 2012; revised January 21, 2012; accepted January 12, 2013. Date of publication May 23, 2013; date of current version September 18, 2013. The work of Z.-P. Jiang was supported by NSF under Grant DMS-0906659 and Grant ECCS-1230040, Recommended by Associate Editor M. Malisoff.

I. Karafyllis is with the Department of Mathematics, National Technical University of Athens, 15780, Athens, Greece (e-mail: iasonkar@central.ntua.gr).

Z.-P. Jiang is with the Department of Electrical and Computer Engineering, Polytechnic Institute of New York University, Brooklyn, NY 11201, USA (e-mail: zjiang@poly.edu).
}

Digital Object Identifier 10.1109/TAC.2013.2264855 first used in [24], where the idea of using a vector Lyapunov function for feedback design purposes was first used. Theorem 3.4 extends the Artstein-Sontag theorem so that vector Lyapunov functions are used and without requiring that all differential inequalities hold at every point of the state space. Therefore, Theorem 3.4 can allow large flexibility for its application. This feature can be crucial for feedback design in large scale systems. Recently, large-scale systems have been studied intensely (see [7], [9], [12], [27]). Section IV of the present work shows that VCLFs can be exploited for the stabilization of large scale systems. Particularly, Corollaries 4.1 and 4.2 provide simple algebraic criteria that allow us to guarantee that a nonlinear system can be globally asymptotically stabilized by means of smooth feedback laws.

The results of the paper are applied to the global stabilization of equilibrium points of (bio)chemical reaction networks taking place in continuous stirred tank reactors (chemostats). Reaction networks have been studied in the past (see the references in [31]) and recent results have provided new insights for their properties (see [1], [4], [31]). Theorem 5.2 provides sufficient conditions for the existence of a smooth stabilizing feedback law for the case that the dilution rate is considered as the control input (the most frequent case in the literature). Our main assumptions on the reaction network hold for biological networks as well. For example, all chemostat models (see [28]) satisfy assumptions (R1), (R2), and (R3) in Section V of this paper. The assumptions are mathematical expressions of the mass balance equations and hold generically for every system which describes the evolution of mass components.

The structure of the present work is as follows. Section II provides the background on vector Lyapunov functions and reviews the recent results in [14], [15]. Section III of the present work contains the definition of the VCLF and the main result of the present work (Theorem 3.4). Section IV is devoted to the derivation of simple sufficient conditions that guarantee the existence of smooth global stabilizers for nonlinear systems (Corollaries 4.1 and 4.2). Finally, the obtained results are applied to reaction networks in Section V. The Appendix contains the proofs of certain auxiliary lemmas needed for the proof of Theorem 3.4.

Notation: Throughout the paper we adopt the following notation:

- Let $I \subseteq \Re_{+}:=[0,+\infty)$ be an interval. By $\mathcal{L}^{\infty}(I ; U)$ (resp. $\mathcal{\mathcal { L }}_{l o c}^{\infty}(I ; U)$ ) we denote the space of measurable and (resp. locally) essentially bounded functions $u(\cdot)$ defined on $I$ and taking values in $U \subseteq \Re^{m}$. For a set $A \subseteq \Re^{n}$, $\operatorname{int}(A)$ denotes the interior of $A \subseteq \Re^{n}$.

- We say that a non-decreasing continuous function $\gamma: \Re_{+} \rightarrow \Re_{+}$is of class $\mathcal{N}_{1}$ if $\gamma(0)=0$. We say that function $\gamma: \Re_{+} \rightarrow \Re_{+}$is positive definite if $\gamma(0)=0$ and $\gamma(s)>0$ for all $s>0$. We say that an increasing continuous function $\gamma: \Re_{+} \rightarrow \Re_{+}$is of class $K$ if $\gamma(0)=0$. We say that an increasing continuous function $\gamma: \Re_{+} \rightarrow \Re_{+}$is of class $K_{\infty}$ if $\gamma(0)=0$ and 
$\lim _{s \rightarrow+\infty} \gamma(s)=+\infty$. By $K L$ we denote the set of all functions $\sigma=\sigma(s, t): \Re^{+} \times \Re^{+} \rightarrow \Re^{+}$with the properties: (i) for each $t \geq 0$ the mapping $\sigma(\cdot, t)$ is of class $K$; (ii) for each $s \geq 0$, the mapping $\sigma(s, \cdot)$ is non-increasing with $\lim _{t \rightarrow+\infty} \sigma(s, t)=0$.

- For every positive integer $j$ and an open, non-empty set $A \subseteq \Re^{n}, C^{j}(A)\left(C^{j}(A ; \Omega)\right)$ denotes the class of functions (taking values in $\Omega \subseteq \Re^{m}$ ) that have continuous derivatives of order $j$ on $A$ and $C^{0}(A ; \Omega)$ denotes the class of continuous functions on $A$, which take values in $\Omega$.

- For a vector $x \in \Re^{n}$, we denote by $x^{\prime}$ its transpose and by $|x|$ its Euclidean norm. $A^{\prime} \in \Re^{n \times m}$ denotes the transpose of the matrix $A \in \Re^{m \times n} . \mathbf{1}_{n}$ denotes the vector $(1, \ldots, 1)^{\prime} \in \operatorname{int}\left(\Re_{+}^{n}\right)$. For a vector $x \in \Re^{n}$, we define $\exp (x)=\left(\exp \left(x_{1}\right), \ldots, \exp \left(x_{n}\right)\right)^{\prime} \in \operatorname{int}\left(\Re_{+}^{n}\right)$.

- For every scalar continuously differentiable function $V$ : $\Re^{n} \rightarrow \Re, \nabla V(x)$ denotes the gradient of $V$ at $x \in \Re^{n}$, i.e., $\nabla V(x)=\left(\partial V / \partial x_{1}(x), \ldots, \partial V / \partial x_{n}(x)\right)$. We say that a function $V: \Re^{n} \rightarrow \Re^{+}$is positive definite if $V(x)>0$ for all $x \neq 0$ and $V(0)=0$. For a vector field $f$ : $\Re^{n} \rightarrow \Re^{n}, L_{f} V(x)=\nabla V(x) f(x)$ denotes the Lie derivative of $V$ along $f$. For a function $\phi: A \rightarrow \Re$, where $A \subseteq \Re^{n}, \operatorname{supp}(\phi)$ denotes the support of $\phi: A \rightarrow \Re$, i.e., $\operatorname{supp}(\phi)=\{x \in A: \phi(x) \neq 0\}$.

\section{BACKGROUND ON VECTOR LYAPUNOV FUNCTIONS}

We consider systems described by Ordinary Differential Equations (ODEs) of the form:

$$
\dot{x}(t)=f(d(t), x(t)), x(t) \in \Re^{n}, d(t) \in D
$$

where $D \subseteq \Re^{l}$ is a non-empty set and $f: D \times \Re^{n} \rightarrow \Re^{n}$ is a continuous mapping with $f(d, 0)=0$ for all $d \in D$ that satisfies the following assumptions:

(A1) There exists a symmetric positive definite matrix $P \in$ $\Re^{n \times n}$ such that for every bounded $S \subset \Re^{n}$, there exists a constant $L \geq 0$ satisfying the following inequality:

$$
\begin{aligned}
& (x-y)^{\prime} P(f(d, x)-f(d, y)) \leq L|x-y|^{2} \\
& \forall x \in S, \forall y \in S, \forall d \in D .
\end{aligned}
$$

(A2) There exists $a \in K_{\infty}$, such that $|f(d, x)| \leq a(|x|)$ for all $(x, d) \in \Re^{n} \times D$.

(A3) There exist functions $h \in C^{1}\left(\Re^{n} ; \Re\right)$ with $h(0)<$ $0, V_{i} \in C^{1}\left(\Re^{n} ; \Re_{+}\right)(i=1, \ldots, m), W \in$ $C^{1}\left(\Re^{n} ; \Re_{+}\right)$being radially unbounded, a function $\delta \in C^{0}\left(\Re_{+} ;(0,+\infty)\right)$, a non-decreasing function $K \in C^{0}\left(\Re_{+} ; \Re_{+}\right), a_{1}, a_{2} \in K_{\infty}$, $\gamma_{i, j} \in \mathcal{N}_{1}, i, j=1, \ldots, m$ with $\gamma_{i, i}(s) \equiv 0$ for $i=1, \ldots, m$ and a family of positive definite functions $\rho_{i} \in C^{0}\left(\Re_{+} ; \Re_{+}\right)(i=1, \ldots, m)$ such that the following inequalities hold:

$$
a_{1}(|x|) \leq \max _{i=1 \ldots . . . m} V_{i}(x) \leq a_{2}(|x|),
$$

for all $x \in \Re^{n}$ satisfying $h(x) \leq 0$.

$$
\sup _{d \in D} \nabla h(x) f(d, x) \leq-\delta(h(x))
$$

for all $x \in \Re^{n}$ satisfying $\quad h(x) \geq 0$.

$$
\sup _{d \in D} \nabla W(x) f(d, x) \leq K(h(x)) W(x),
$$

for all $x \in \Re^{n}$ satisfying $h(x) \geq 0$.
Moreover, for every $i=1, \ldots, m$ and $x \in \Re^{n}$ with $h(x) \leq 0$, the following implication holds:

$$
\begin{aligned}
& \text { "If } \max _{j=1, \ldots, m} \gamma_{i, j}\left(V_{j}(x)\right) \leq V_{i}(x), \\
& \text { then } \sup _{d \in D} \nabla V_{i}(x) f(d, x) \leq-\rho_{i}\left(V_{i}(x)\right) " .
\end{aligned}
$$

Theorem 2.7 in [15]: Consider system (1) under assumptions (A1-3). If the following set of small-gain conditions holds for each $r=2, \ldots, m$ :

$$
\left(\gamma_{i_{1}, i_{2}} \circ \gamma_{i_{2}, i_{3}} \circ \cdots \circ \gamma_{i_{r}, i_{1}}\right)(s)<s, \forall s>0
$$

for all $i_{j} \in\{1, \ldots, m\}, i_{j} \neq i_{l}$ if $j \neq l$, then system (1) is Robustly Globally Asymptotically Stable (RGAS). That is, there exists $\sigma \in K L$ such that for every $d \in \mathcal{L}_{\text {loc }}^{\infty}\left(\Re_{+} ; D\right), x_{0} \in$ $\Re^{n}$ the solution $x(t) \in \Re^{n}$ of $(1)$ with $x(0)=x_{0}$ corresponding to $d \in \mathcal{L}_{\text {loc }}^{\infty}\left(\Re_{+} ; D\right)$ satisfies $|x(t)| \leq \sigma\left(\left|x_{0}\right|, t\right)$, for all $t \geq 0$.

1) Discussion and Explanations: (a) If implications (5) hold for all $i=1, \ldots, m$ and $x \in \Re^{n}$, then one simply takes $h(x) \equiv-1$ and arbitrary functions $W \in C^{1}\left(\Re^{n} ; \Re_{+}\right)$, $\delta \in C^{0}\left(\Re_{+} ;(0,+\infty)\right), K \in C^{0}\left(\Re_{+} ; \Re_{+}\right)$. In this way we obtain Corollary 4.2 in [14]. Therefore the difference between Theorem 2.7 in [15] and Corollary 4.2 in [14] is that Theorem 2.7 assumes that the Lyapunov differential inequalities (5) hold only for a certain region of the state space (at the cost of the additional inequalities (3), (4)). However, Corollary 4.2 in [14] allows the study of time-varying systems as well. (b) Notice that $W \in C^{1}\left(\Re^{n} ; \Re_{+}\right)$is not necessarily positive definite. (c) Theorem 2.7 in [15] is remarkably different from other vector Lyapunov results (see [3], [8], [18], [19], [23]). The vector Lyapunov results in [3], [8], [18], [19], [23] assume that the Lyapunov differential inequalities are valid on the whole state space while assumption (A3) requires that each one of the differential inequalities (5) is valid only for a limited region of the state space (described by the inequalities $h(x) \leq 0$ and $\left.\max _{j=1, \ldots, m} \gamma_{i, j}\left(V_{j}(x)\right) \leq V_{i}(x)\right)$. Moreover, the form of inequalities (5) is extremely simple and very similar to the differential inequality used for the single Lyapunov function. All these features of Theorem 2.7 in [15] are exploited in the following section.

\section{Vector Robust CONTROL LyAPunOv FunCtions}

Consider the feedback stabilization problem for the system:

$$
\begin{aligned}
& \dot{x}(t)=f(d(t), x(t))+g(x(t)) u(t) \\
& x(t) \in \Re^{n}, u(t) \in U \subseteq \Re, d(t) \in D
\end{aligned}
$$

where $D \subseteq \Re^{l}$ is compact and $f: D \times \Re^{n} \rightarrow \Re^{n}, g: \Re^{n} \rightarrow \Re^{n}$ are continuous mappings with $f(d, 0)=0$ for all $d \in D$ that satisfy the following assumption:

(H) There exists a symmetric positive definite matrix $P \in$ $\Re^{n \times n}$ such that for every bounded $S \subset \Re^{n} \times \Re$, there exists a constant $L \geq 0$ satisfying the following inequality:

$$
\begin{aligned}
& (x-y)^{\prime} P(f(d, x)+g(x) u-f(d, y)-g(y) u) \\
& \quad \leq L|x-y|^{2} \\
& \forall(x, u, y, u) \in S \times S, \forall d \in D .
\end{aligned}
$$


For the control set $U \subset \Re$ we consider the following three cases:

(P1) $U=\Re$.

(P2) There exists a constant $a \geq 0$ such that $U=[-a,+\infty)$.

(P3) There exist two constants $a, b \geq 0$ such that $U=$ $[-a, b]$.

We next proceed to the definition of the Vector Robust Control Lyapunov Function (VRCLF) for system (7).

Definition 3.1: Consider system (7) under assumptions $(\mathrm{H})$, (P1). Suppose that there exist functions $\eta \in C^{1}\left(\Re^{n} ; \Re\right)$ with $\eta(0)<0, V_{i} \in C^{1}\left(\Re^{n} ; \Re_{+}\right)(i=1, \ldots, m)$, $W \in C^{1}\left(\Re^{n} ;[1,+\infty)\right)$ being radially unbounded, a function $\delta \in C^{0}\left(\Re_{+} ;(0,+\infty)\right)$, a non-decreasing function $K \in C^{0}\left(\Re_{+} ;[1,+\infty)\right), \gamma_{i, j} \in \mathcal{N}_{1}, i, j=1, \ldots, m$, with $\gamma_{i, i}(s) \equiv 0$ for $i=1, \ldots, m$, a positive definite function $\rho \in C^{0}\left(\Re_{+} ; \Re_{+}\right)$and a constant $\varepsilon>0$ such that the following properties hold:

(i) There exist functions $a_{1}, a_{2} \in K_{\infty}$ such that the following inequality holds for all $x \in \Re^{n}$ :

$$
a_{1}(|x|) \leq \max _{i=1, \ldots, m} V_{i}(x) \leq a_{2}(|x|) .
$$

(ii) The following implications hold for all $x \in \Re^{n}$ with $\eta(x) \leq \varepsilon:$

"If $\max _{j=1, \ldots, m} \gamma_{i, j}\left(V_{j}(x)\right) \leq V_{i}(x)$ and $L_{g} V_{i}(x)=0$, then $F_{i}(x) \leq 0 . "$

"If $\max _{s=1, \ldots, m} \gamma_{i, s}\left(V_{s}(x)\right) \leq V_{i}(x)$,

$\max _{s=1, \ldots, m} \gamma_{j, s}\left(V_{s}(x)\right) \leq V_{j}(x)$

and $L_{g} V_{i}(x) L_{g} V_{j}(x)<0$, then $F_{i}(x) \leq \frac{L_{g} V_{i}(x)}{L_{g} V_{j}(x)} F_{j}(x)$."

where $F_{i}(x):=\max _{d \in D}\left(\nabla V_{i}(x) f(d, x)\right)+\rho\left(V_{i}(x)\right), i=$ $1, \ldots, m$.

(iii) The following implications hold for all $x \in \Re^{n}$ satisfying $\eta(x) \geq 0$ :

"If $L_{g} \eta(x)=0$, then $H(x) \leq 0 . "$

"If $L_{g} W(x)=0$, then $R(x) \leq 0$."

"If $L_{g} \eta(x) L_{g} W(x)<0$, then $H(x) \leq \frac{L_{g} \eta(x)}{L_{g} W(x)} R(x)$."

where $H(x):=\max _{d \in D}(\nabla \eta(x) f(d, x))+\delta(\eta(x)), R(x):=$ $\max _{d \in D}(\nabla W(x) f(d, x))-K(\eta(x)) W(x)$.

(iv) The following implications hold for all $x \in \Re^{n}$ with $0 \leq$ $\eta(x) \leq \varepsilon:$

"If $\max _{s=1, \ldots, m} \gamma_{j, s}\left(V_{s}(x)\right) \leq V_{j}(x)$ and $L_{g} \eta(x) L_{g} V_{j}(x)<0$,

$$
\text { then } H(x) \leq \frac{L_{g} \eta(x)}{L_{g} V_{j}(x)} F_{j}(x) . "
$$

"If $\max _{s=1, \ldots, m} \gamma_{j, s}\left(V_{s}(x)\right) \leq V_{j}(x)$ and $L_{g} W(x) L_{g} V_{j}(x)<0$,

$$
\text { then } R(x) \leq \frac{L_{g} W(x) F_{j}(x)}{L_{g} V_{j}(x)} "
$$

(v) The small-gain conditions (6) hold.

(vi) There exist an open set $A \subseteq \Re^{n}$ with $0 \in A$ and a locally Lipschitz function $k \in C^{\nu}(A ; U)$, where $\nu \in$
$\{0,1,2, \ldots\} \cup\{\infty\}$, with $k(0)=0$, such that the following implication holds for all $i=1, \ldots, m$ :

$$
\text { "If } \max _{j=1, \ldots, m} \gamma_{i, j}\left(V_{j}(x)\right) \leq V_{i}(x) \text { and } x \in A,
$$

then $F_{i}(x)+L_{g} V_{i}(x) k(x) \leq 0 "$.

Then, the family of functions $V_{i} \in C^{1}\left(\Re^{n} ; \Re_{+}\right)(i=$ $1, \ldots, m)$ is called a Vector Robust Control Lyapunov Function (VRCLF) for system (7) under assumptions (H), (P1) or we say that system (7) under assumptions (H), (P1) admits the VRCLF $V_{i} \in C^{1}\left(\Re^{n} ; \Re_{+}\right)(i=1, \ldots, m)$ with gains $\gamma_{i, j} \in \mathcal{N}_{1}(i, j=1, \ldots, m)$ and auxiliary functions $\eta, W$

Definition 3.2: Consider system (7) under assumptions (H), (P2). Suppose that there exist functions $\eta \in C^{1}\left(\Re^{n} ; \Re\right)$ with $\eta(0)<0, V_{i} \in C^{1}\left(\Re^{n} ; \Re_{+}\right)(i=1, \ldots, m)$, $W \in C^{1}\left(\Re^{n} ;[1,+\infty)\right)$ being radially unbounded, a function $\delta \in C^{0}\left(\Re_{+} ;(0,+\infty)\right)$, a non-decreasing function $K \in C^{0}\left(\Re_{+} ;[1,+\infty)\right), \gamma_{i, j} \in \mathcal{N}_{1}, i, j=1, \ldots, m$, with $\gamma_{i, i}(s) \equiv 0$ for $i=1, \ldots, m$, a positive definite function $\rho \in C^{0}\left(\Re_{+} ; \Re_{+}\right)$and a constant $\varepsilon>0$ such that properties (i)-(vi) hold. Moreover, assume that the following property holds:

(vii)The following implications hold:

"If $\max _{s=1, \ldots, m} \gamma_{i, s}\left(V_{s}(x)\right) \leq V_{i}(x), \eta(x) \leq \varepsilon$ and $L_{g} V_{i}(x)>0$, then $F_{i}(x)-a L_{g} V_{i}(x)<0 . "$

"If $\eta(x) \geq 0, L_{g} \eta(x)>0$, then $H(x)-a L_{g} \eta(x)<0$."

"If $\eta(x) \geq 0, L_{g} W(x)>0$, then $R(x)-a L_{g} W(x)<0$ ".

Then, the family of functions $V_{i} \in C^{1}\left(\Re^{n} ; \Re_{+}\right)(i=$ $1, \ldots, m)$ is called a VRCLF for system (7) or we say that system (7) under assumptions (H), (P2) admits the VRCLF $V_{i} \in C^{1}\left(\Re^{n} ; \Re_{+}\right)(i=1, \ldots, m)$ with gains $\gamma_{i, j} \in \mathcal{N}_{1}(i, j=1, \ldots, m)$ and auxiliary functions $\eta, W$.

Definition 3.3: Consider system (7) under assumptions (H), (P3). Suppose that there exist functions $\eta \in C^{1}\left(\Re^{n} ; \Re\right)$ with $\eta(0)<0, V_{i} \in C^{1}\left(\Re^{n} ; \Re_{+}\right)(i=1, \ldots, m)$, $W \in C^{1}\left(\Re^{n} ;[1,+\infty)\right)$ being radially unbounded, a function $\delta \in C^{0}\left(\Re_{+} ;(0,+\infty)\right)$, a non-decreasing function $K \in C^{0}\left(\Re_{+} ;[1,+\infty)\right), \gamma_{i, j} \in \mathcal{N}_{1}, i, j=1, \ldots, m$, with $\gamma_{i, i}(s) \equiv 0$ for $i=1, \ldots, m$, a positive definite function $\rho \in C^{0}\left(\Re_{+} ; \Re_{+}\right)$and a constant $\varepsilon>0$ such that properties (i)-(vii) hold. Moreover, assume that the following property holds:

(viii) The following implications hold:

$$
\begin{aligned}
& \text { "If } \max _{s=1, \ldots, m} \gamma_{i, s}\left(V_{s}(x)\right) \leq V_{i}(x), \eta(x) \leq \varepsilon \text { and } L_{g} V_{i}(x)<0, \\
& \text { then } F_{i}(x)+b L_{g} V_{i}(x)<0 . " \\
& \text { "If } \eta(x) \geq 0, L_{g} \eta(x)<0 \text {, then } H(x)+b L_{g} \eta(x)<0 " .
\end{aligned}
$$

"If $\eta(x) \geq 0, L_{g} W(x)<0$, then $R(x)+b L_{g} W(x)<0$ ".

Then, the family of functions $V_{i} \in C^{1}\left(\Re^{n} ; \Re_{+}\right)(i=1, \ldots, m)$ is called a VRCLF for system (7) or we say that system (7) under assumptions (H), (P3) admits the VRCLF $V_{i} \in C^{1}\left(\Re^{n} ; \Re_{+}\right)(i=1, \ldots, m)$ with gains $\gamma_{i, j} \in \mathcal{N}_{1}$ and auxiliary functions $\eta, W$. 
We are now ready to state the main result of the section. Its proof is given in the Appendix.

Theorem 3.4: Consider system (7) under assumption $(\mathrm{H})$ and under one of the assumptions (P1), (P2), (P3). Suppose that system (7) admits the VRCLF $V_{i} \in C^{1}\left(\Re^{n} ; \Re_{+}\right)(i=$ $1, \ldots, m)$ with gains $\gamma_{i, j} \in \mathcal{N}_{1}(i, j=1, \ldots, m)$ and auxiliary functions $\eta, W$. Then, there exists a locally Lipschitz function $\tilde{k} \in C^{\nu}\left(\Re^{n} ; U\right) \cap C^{\infty}\left(\Re^{n} \backslash\{0\} ; U\right)$, where $\nu \in\{0,1,2, \ldots\} \cup\{\infty\}$ is the number involved in property (vi) of the definition of the VRCLF, with $\tilde{k}(0)=0$, such that the closed-loop system (7) with $u=\tilde{k}(x)$ is RGAS.

Here, it should be noted that the existence of a VRCLF is a necessary condition as well for the existence of a smooth globally stabilizing feedback if the vector fields $f: D \times \Re^{n} \rightarrow \Re^{n}$, $g: \Re^{n} \rightarrow \Re^{n}$ are locally Lipschitz. More specifically, if there exists a locally Lipschitz function $k \in C^{\nu}(A ; U)$, where $\nu \in$ $\{0,1,2, \ldots\} \cup\{\infty\}$, with $k(0)=0$, such that the closed-loop system (7) with $u=k(x)$ is RGAS and if the vector fields $f: D \times \Re^{n} \rightarrow \Re^{n}, g: \Re^{n} \rightarrow \Re^{n}$ are locally Lipschitz, then the converse Lyapunov theorem in [20] guarantees the existence of a positive definite and radially unbounded function $V_{1} \in$ $C^{\infty}\left(\Re^{n} ; \Re_{+}\right)$and a positive definite function $\rho \in C^{0}\left(\Re_{+} ; \Re_{+}\right)$ with $\max _{d \in D}\left(\nabla V_{1}(x) f(d, x)\right)+L_{g} V_{1}(x) k(x)+\rho\left(V_{1}(x)\right) \leq 0$ for all $x \in \Re^{n}$. All properties of Definitions $3.1,3.2$ or 3.3 with $m=1$ are direct consequences of the above fact.

The proofs of Theorem 3.4 and Theorem 2.7 in [15] show that the solution of the closed-loop system (7) with $u=\tilde{k}(x)$ reaches the set $\left\{x \in \Re^{n}: 5 \eta(x) \leq 2 \varepsilon\right\}$ in finite time. Therefore, the set $\left\{x \in \Re^{n}: 5 \eta(x) \leq 2 \varepsilon\right\}$ is important: the solution of the closed-loop system evolves in this set after an initial transient period and consequently the controller performance is affected by the choice of both $\eta(x)$ and $\varepsilon>0$. A trade-off can arise: a good performance of the controller can be achieved by making the set $\left\{x \in \Re^{n}: 5 \eta(x) \leq 2 \varepsilon\right\}$ sufficienly "small". However, a "small" set $\left\{x \in \Re^{n}: \overline{5} \eta(x) \leq 2 \varepsilon\right\}$ means a large transient period during which we have no guarantee of performance.

For the proof of Theorem 3.4 we need two technical lemmas. Their proofs are given in the Appendix. The first lemma deals with a set of inequalities.

Lemma 3.5: Let $a, b \geq 0$ with $a+b>0, f_{i}, g_{i} \in \Re(i=$ $1, \ldots, m)$ be given constants. There exists $u \in \Re$ such that $f_{i}+g_{i} u<0$, for all $i=1, \ldots, m$ if and only if the following implications hold:

(I) If $g_{i}=0$ for certain $i \in\{1, \ldots, m\}$, then $f_{i}<0$,

(II) If $g_{i} g_{j}<0$ for certain pair $(i, j) \in\{1, \ldots, m\} \times$ $\{1, \ldots, m\}$, then $f_{i}<g_{i} f_{j} / g_{j}$.

Moreover, there exists $u \in(-a,+\infty)$ such that $f_{i}+g_{i} u<0$, for all $i=1, \ldots, m$ if and only if implications (I), (II) hold and the following implication holds as well:

(III) If $g_{i}>0$ for certain $i \in\{1, \ldots, m\}$, then $f_{i}-a g_{i}<0$.

Finally, there exists $u \in(-a, b)$ such that $f_{i}+g_{i} u<0$, for all $i=1, \ldots, m$ if and only if implications (I), (II), (III) hold and the following implication holds as well:

(IV) If $g_{i}<0$ for certain $i \in\{1, \ldots, m\}$, then $f_{i}+b g_{i}<0$.

The second technical lemma guarantees that we may assume that all requirements (i)-(vi) for a VRCLF hold for functions $\gamma_{i, j} \mathcal{N}_{1}$, which are positive definite for $i \neq j$.

Lemma 3.6: Suppose that system (7) admits the VRCLF $V_{i} \in$ $C^{1}\left(\Re^{n} ; \Re_{+}\right)(i=1, \ldots, m)$ with gains $\gamma_{i, j} \in \mathcal{N}_{1}(i, j=$ $1, \ldots, m)$ and auxiliary functions $\eta, W$. Then all properties of the definition of the VRCLF hold with functions $\gamma_{i, j} \in \mathcal{N}_{1}$, $i, j=1, \ldots, m$, which are positive definite for $i \neq j$ and satisfy $\lim _{\rightarrow+\infty} \gamma_{i, j}(s)=+\infty$ for all $i, j=1, \ldots, m$ with $i \neq j$.

It should be noted that the proof of Theorem 3.4 is based on partition of unity arguments. A different way of proving Theorem 3.4 is by using Michael's theorem. However, the use of Michael's theorem results in a continuous feedback (instead of a smooth feedback law).

\section{Application to FeEdBack Stabilizer Design}

A natural question of practical importance is how to construct a VRCLF which satisfies the (involved) assumptions of the VRCLF. The purpose of this section is to provide conditions that allow us to use simple VRCLFs (namely, the functions $V_{i}(x)=x_{i}^{2} / 2$ for $\left.i=1, \ldots, n\right)$. More specifically, this section is devoted to the feedback stabilization problem for nonlinear systems of the form:

$$
\dot{x}_{i}(t)=f_{i}(x(t))+g_{i}(x(t)) u(t), \quad i=1, . ., n
$$

where $x(t)=\left(x_{1}(t), \ldots, x_{n}(t)\right)^{\prime} \in \Re^{n}, u(t) \in U \subseteq \Re$ and the mappings $f_{i}: \Re^{n} \longrightarrow \Re, g_{i}: \Re^{n} \rightarrow \Re$ are locally Lipschitz with $f_{i}(0)=0$ for $i=1, \ldots, n$. More specifically, we use the results of the previous section in order to derive sufficient conditions for the existence of a globally stabilizing feedback. Interestingly, the developed sufficient conditions are easily checkable even for large $n$ (large scale systems). Our main results are the following corollaries.

Corollary 4.1: Consider system (23) under assumption (P1) and suppose that there exist functions $\eta \in C^{1}\left(\Re^{n} ; \Re\right)$ with $\eta(0)<0, W \in C^{1}\left(\Re^{n} ;[1,+\infty)\right)$ being radially unbounded, a function $\delta \in C^{0}\left(\Re_{+} ;(0,+\infty)\right)$, a non-decreasing function $K \in C^{0}\left(\Re_{+} ;[1,+\infty)\right), \tilde{\gamma}_{i, j} \in \mathcal{N}_{1}, i, j=1, \ldots, n$, with $\tilde{\gamma}_{i, i}(s) \equiv 0$ for $i=1, \ldots, n$ which satisfy the small-gain conditions (6), a function $Q \in C^{0}\left(\Re ; \Re_{+}\right)$with $Q(x)>0$ for all $x \neq 0$ and a constant $\varepsilon>0$ such that the following implications hold for all $i, j \in\{1, \ldots, n\}$ :

"If $\max _{s=1, \ldots, n} \tilde{\gamma}_{i, s}\left(\left|x_{s}\right|\right) \leq\left|x_{i}\right|$.

$\max _{s=1, \ldots, n} \tilde{\gamma}_{j, s}\left(\left|x_{s}\right|\right) \leq\left|x_{j}\right|, \eta(x) \leq \varepsilon$ and

$x_{i} x_{j} g_{i}(x) g_{j}(x)<0$, then

$\frac{f_{i}(x) g_{j}(x)-f_{j}(x) g_{i}(x)}{x_{i} Q\left(x_{i}\right) g_{j}(x)-x_{j} Q\left(x_{j}\right) g_{i}(x)} \leq-1 "$.

"If $g_{i}(x)=0, \eta(x) \leq \varepsilon$ and $\max _{s=1 \ldots, n} \tilde{\gamma}_{i, s}\left(\left|x_{s}\right|\right) \leq\left|x_{i}\right|$,

then $x_{i} f_{i}\left(x_{i}\right)+x_{i}^{2} Q\left(x_{i}\right) \leq 0 "$.

"If $\max _{s=1, \ldots, n} \tilde{\gamma}_{j, s}\left(\left|x_{s}\right|\right) \leq\left|x_{j}\right|, 0 \leq \eta(x) \leq \varepsilon$ and

$x_{j} g_{j}(x) \sum_{l=1}^{n} \frac{\partial \eta}{\partial x_{l}}(x) g_{l}(x)<0$, then

$\sum_{l=1}^{n} \frac{\partial \eta}{\partial x_{l}}(x)\left(f_{l}(x)-g_{l}(x) \frac{f_{j}(x)+x_{j} Q\left(x_{j}\right)}{g_{j}(x)}\right)$

$\leq-\delta(\eta(x)) "$.

"If $\max _{s=1, \ldots, n} \tilde{\gamma}_{j, s}\left(\left|x_{s}\right|\right) \leq\left|x_{j}\right|, 0 \leq \eta(x) \leq \varepsilon$ and

$x_{j} g_{j}(x) \sum_{l=1}^{n} \frac{\partial W}{\partial x_{l}}(x) g_{l}(x)<0$, then

$\sum_{l=1}^{n} \frac{\partial W}{\partial x_{l}}(x)\left(f_{l}(x)-g_{l}(x) \frac{f_{j}(x)+x_{j} Q\left(x_{j}\right)}{g_{j}(x)}\right)$

$\leq K(\eta(x)) W(x) "$. 
Moreover, suppose that there exists a vector $k \in \Re^{n}$ such that $x_{i} f_{i}(x)+x_{i} g_{i}(x) k^{\prime} x \leq-x_{i}^{2} Q\left(x_{i}\right)$ for all $x=\left(x_{1}, \ldots, x_{n}\right)^{\prime} \in \Re^{n}$ in a neighborhood of $0 \in \Re^{n}$ with $\max _{s=1, \ldots, n} \tilde{\gamma}_{i, s}\left(\left|x_{s}\right|\right) \leq\left|x_{i}\right|(i=1, \ldots, n)$. Finally, suppose that there exists a function $\bar{k} \in C^{0}\left(\Re^{n} ; \Re\right)$ such that $\sum_{i=1}^{n} \partial W / \partial x_{i}(x)\left(f_{i}(x)+g_{i}(x) \bar{k}(x)\right) \leq K(\eta(x)) W(x)$ and $\sum_{i=1}^{n} \partial \eta / \partial x_{i}(x)\left(f_{i}(x)+g_{i}(x) \bar{k}(x)\right) \leq-\delta(\eta(x))$ for all $x \in \Re^{n}$ with $\eta(x) \geq 0$. Then there exists $\tilde{k} \in C^{\infty}\left(\Re^{n} ; \Re\right)$, with $\tilde{k}(0)=0$, such that $0 \in \Re^{n}$ is GAS for the closed-loop system $\dot{x}_{i}(t)=f_{i}(x(t))+g_{i}(x(t)) \tilde{k}(x(t)), i=1, \ldots, n$

Corollary 4.2: Consider system (23) under assumption (P3) with $a, b>0$ and suppose that there exist functions $\eta \in C^{1}\left(\Re^{n} ; \Re\right)$ with $\eta(0)<0, W \in C^{1}\left(\Re^{n} ;[1,+\infty)\right)$ being radially unbounded, a function $\delta \in C^{0}\left(\Re_{+} ;(0,+\infty)\right)$, a non-decreasing function $K \in C^{0}\left(\Re_{+} ;[1,+\infty)\right), \tilde{\gamma}_{i, j} \in \mathcal{N}_{1}$, $i, j=1, \ldots, n$, with $\tilde{\gamma}_{i, i}(s) \equiv 0$ for $i=1, \ldots, n$ which satisfy the small-gain conditions (6), a function $Q \in C^{0}\left(\Re ; \Re_{+}\right)$ with $Q(x)>0$ for all $x \neq 0$ and a constant $\varepsilon>0$ such that implications (24), (25), (26) and (27) hold for all $i, j \in\{1, \ldots, n\}$. Moreover, suppose that there exists a vector $k \in \Re^{n}$ such that $x_{i} f_{i}(x)+x_{i} g_{i}(x) k^{\prime} x \leq-x_{i}^{2} Q\left(x_{i}\right)$ for all $x=\left(x_{1}, \ldots, x_{n}\right)^{\prime} \in \Re^{n}$ in a neighborhood of $0 \in \Re^{n}$ with $\max _{s=1, \ldots, n} \tilde{\gamma}_{i, s}\left(\left|x_{s}\right|\right) \leq\left|x_{i}\right|(i=1, \ldots, n)$ and suppose that there exists a function $\bar{k} \in C^{0}\left(\Re^{n} ; U\right)$ such that $\sum_{i=1}^{n} \partial W / \partial x_{i}(x)\left(f_{i}(x)+g_{i}(x) \bar{k}(x)\right) \leq K(\eta(x)) W(x)$ and $\sum_{i=1}^{n} \partial \eta / \partial x_{i}(x)\left(f_{i}(x)+g_{i}(x) \bar{k}(x)\right) \leq-\delta(\eta(x))$ for all $x \in \Re^{n}$ with $\eta(x) \geq 0$. Finally, suppose that the following implications hold:

$$
\begin{aligned}
& \text { "If } \max _{s=1, \ldots, n} \tilde{\gamma}_{i, s}\left(\left|x_{s}\right|\right) \leq\left|x_{i}\right|, \eta(x) \leq \varepsilon \text { and } x_{i} g_{i}(x)>0, \\
& \text { then } x_{i} f_{i}(x)+x_{i}^{2} Q\left(x_{i}\right)-a x_{i} g_{i}(x)<0 . " \\
& \text { "If } \max _{s=1, \ldots, n} \tilde{\gamma}_{i, s}\left(\left|x_{s}\right|\right) \leq\left|x_{i}\right|, \eta(x) \leq \varepsilon \text { and } x_{i} g_{i}(x)<0, \\
& \text { then } x_{i} f_{i}(x)+x_{i}^{2} Q\left(x_{i}\right)+b x_{i} g_{i}(x)<0 " .
\end{aligned}
$$

Then there exists $\tilde{k} \in C^{\infty}\left(\Re^{n} ; U\right)$, with $\tilde{k}(0)=0$, such that $0 \in \Re^{n}$ is GAS for the closed-loop system $\dot{x}_{i}(t)=f_{i}(x(t))+$ $g_{i}(x(t)) \tilde{k}(x(t)), i=1, . ., n$

Proofs of Corollary 4.1 and Corollary 4.2: A direct application of Theorem 3.4 with $m=n, V_{i}(x)=x_{i}^{2} / 2$ for $i=1, \ldots, n, \gamma_{i, j}(s):=\left(\tilde{\gamma}_{i, j}(\sqrt{2 s})\right)^{2} / 2$ for $i, j=1, \ldots, n$ and $\rho(s):=2 s \min (Q(\sqrt{2 s}), Q(-\sqrt{2 s}))$, $a_{1}(s):=s^{2} /(2 n), a_{2}(s):=s^{2} / 2$ for all $s \geq 0$. Notice that implications (9), (10) are directly implied by implications (25), (24), respectively and the above definitions. Implications (11), (12), (13) are direct consequences of the existence of a function $\bar{k} \in C^{0}\left(\Re^{n} ; U\right)$ such that $\sum_{i=1}^{n} \partial W / \partial x_{i}(x)\left(f_{i}(x)+g_{i}(x) \bar{k}(x)\right) \leq K(\eta(x)) W(x)$ and $\sum_{i=1}^{n} \partial \eta / \partial x_{i}(x)\left(f_{i}(x)+g_{i}(x) \bar{k}(x)\right) \leq-\delta(\eta(x))$ for all $x \in \Re^{n}$ with $\eta(x) \geq 0$

For Corollary 4.2, we notice that (17), (20) are equivalent to implications (28), (29) and that implications (18), (19), (21), (22) are directly implied by the inequalities $\sum_{i=1}^{n} \partial W / \partial x_{i}(x)\left(f_{i}(x)+g_{i}(x) \bar{k}(x)\right) \leq K(\eta(x)) W(x)$ and $\sum_{i=1}^{n} \partial \eta / \partial x_{i}(x)\left(f_{i}(x)+g_{i}(x) \bar{k}(x)\right) \leq-\delta(\eta(x))$ for all $x \in \Re^{n}$ with $\eta(x) \geq 0$ (possibly by replacing $K(\eta(x))$ and $\delta(\eta(x))$ by $2 K(\eta(x))$ and $\delta(\eta(x)) / 2$, respectively) and the fact that $\bar{k}(x) \in U$ for all $x \in \Re^{n}$. Notice that since $a, b>0$, property (vi) of Definition 3.2 holds with the linear feedback law $u=k^{\prime} x$ (possibly by replacing the initial neighborhood by another neighborhood with $|k||x| \leq \min (a, b))$. The proof is complete.

A direct application of Corollary 4.1 and Corollary 4.2 is obtained by selecting $\eta(x) \equiv-1, \varepsilon=1$ : in this case implications (26), (27) are automatically satisfied for arbitrary functions $W, \delta, K$. Moreover, the assumption of the existence of a function $\bar{k} \in C^{0}\left(\Re^{n} ; U\right)$ such that $\sum_{i=1}^{n} \partial W / \partial x_{i}(x)\left(f_{i}(x)+g_{i}(x) \bar{k}(x)\right) \leq K(\eta(x)) W(x)$ and $\sum_{i=1}^{n} \partial \eta / \partial x_{i}(x)\left(f_{i}(x)+g_{i}(x) \bar{k}(x)\right) \leq-\delta(\eta(x))$ for all $x \in \Re^{n}$ with $\eta(x) \geq 0$ is automatically satisfied for arbitrary $\bar{k} \in C^{0}\left(\Re^{n} ; U\right)$. The reader should notice that implications (24), (25) are easily checkable: the gain functions $\tilde{\gamma}_{i, j} \in \mathcal{N}_{1}$, $i, j=1, \ldots, n$ are selected so that implications (24), (25) hold. The following example shows how to use Corollary 4.1 with $\eta(x) \equiv-1, \varepsilon=1$ for a nonlinear system.

Example 4.3: Consider the nonlinear system

$$
\begin{aligned}
\dot{x}_{1}(t) & =-x_{1}(t)+x_{2}(t) \\
\dot{x}_{2}(t) & =-x_{2}(t)+g(x(t)) u(t) \\
\dot{x}_{3}(t) & =x_{1}^{2}(t)+u(t) \\
x(t) & =\left(x_{1}(t), x_{2}(t), x_{3}(t)\right)^{\prime} \in \Re^{3}, u(t) \in \Re
\end{aligned}
$$

where $g: \Re^{3} \rightarrow \Re$ is a locally Lipschitz function. The problem that we study in this example is:

(Q) "For what functions $g: \Re^{3} \rightarrow \Re$, system (30) can be stabilized globally by a smooth feedback?"

Using the function $V(x)=\left(x_{1}^{2}+p x_{2}^{2}+q x_{3}^{2}\right) / 2$ as a CLF candidate, where $p, q>0$, we conclude that this function is a CLF (in the sense explained in [29]) for system (30) provided that the following condition holds:

$$
\begin{aligned}
& \text { For each } x \in \Re^{3} \backslash\{0\} \text { satisfying } q x_{3}=-p x_{2} g(x) \\
& \text { it holds that } x_{1}^{2}-x_{1} x_{2}+p x_{2}^{2}>q x_{1}^{2} x_{3} .
\end{aligned}
$$

Therefore, using the results in [29], we are in a position to guarantee that there exists an "almost smooth" feedback law that globally stabilizes (30), provided that (31) holds and the "small-control" property holds. Here, we obtain different conditions for the function $g: \Re^{3} \rightarrow \Re$, which allow smooth stabilizability of system (30). We show that system (30) can be stabilized globally by smooth feedback provided that there exist constants $\lambda \in(0,1), \sigma \in(0,1)$, functions $\gamma \in K_{\infty} \cap C^{1}\left(\Re_{+}\right)$ with $d \gamma / d s(0)>\sigma|g(0)| / 1-\sigma, Q \in C^{0}\left(\Re ; \Re_{+}\right)$with $0<$ $Q(x) \leq \sigma$ for all $x \neq 0$, such that the locally Lipschitz function $g: \Re^{3} \rightarrow \Re$ satisfies the following implication:

$$
\begin{aligned}
& \lambda(1-\sigma)\left|x_{1}\right| \leq \gamma\left(\left|x_{3}\right|\right), \lambda\left|x_{2}\right| \leq \gamma\left(\left|x_{3}\right|\right) \leq\left|x_{2}\right|, \\
& x_{2} x_{3} g(x)<0 \Rightarrow x_{2}^{2}+x_{2} x_{1}^{2} g(x) \\
& \quad \geq\left|x_{2} x_{3} g(x)\right| Q\left(x_{3}\right)+x_{2}^{2} Q\left(x_{2}\right) .
\end{aligned}
$$

In order to show the qualitative difference of conditions (31) and (32) notice that the locally Lipschitz function $g(x):=$ $x_{2} x_{3}\left(\left|x_{2}\right|-\gamma\left(\left|x_{3}\right|\right)\right)\left(\gamma\left(\left|x_{3}\right|\right)-\lambda\left|x_{2}\right|\right)$ satisfies condition (32) for arbitrary selection of $\lambda \in(0,1)$ and $\gamma \in K_{\infty} \cap C^{1}\left(\Re_{+}\right)$ with $d \gamma / d s(0)>0$. On the other hand, the function $g(x):=x_{2} x_{3}\left(\left|x_{2}\right|-\gamma\left(\left|x_{3}\right|\right)\right)\left(\gamma\left(\left|x_{3}\right|\right)-\lambda\left|x_{2}\right|\right)$ does not satisfy condition (31) for any choice of $p, q>0$. To see this, notice that the equation $q x_{3}=-p x_{2} g(x)$ holds for $x_{2} \neq 0$ and $x_{3}=$ $\gamma^{-1}\left((\lambda+1) 2^{-1}\left|x_{2}\right|+2^{-1} \sqrt{(\lambda-1)^{2} x_{2}^{2}+4 q p^{-1} x_{2}^{-2}}\right)$.

However, the inequality $x_{1}^{2}-x_{1} x_{2}+p x_{2}^{2}>q x_{1}^{2} x_{3}$ cannot be satisfied for arbitrary $x_{1}=1, x_{2} \neq 0$ and $x_{3}=$ 
$\gamma^{-1}\left((\lambda+1) 2^{-1}\left|x_{2}\right|+2^{-1} \sqrt{(\lambda-1)^{2} x_{2}^{2}+4 q p^{-1} x_{2}^{-2}}\right)$

(notice that if $x_{2} \rightarrow 0$ then $x_{3}=\gamma^{-1}\left((\lambda+1) 2^{-1}\left|x_{2}\right|+2^{-1}\right.$ $\left.\left.\sqrt{(\lambda-1)^{2} x_{2}^{2}+4 q p^{-1} x_{2}^{-2}}\right) \rightarrow+\infty\right)$.

In order to obtain condition (32), we apply Corollary 4.1 with $\eta(x) \equiv-1, \varepsilon=-1, Q \in C^{0}\left(\Re ; \Re_{+}\right)$satisfying $0<Q(x) \leq \sigma$ for all $x \neq 0$, where $\sigma \in(0,1)$. We first notice that implications (25) and the small-gain conditions (6) hold for arbitrary $\gamma \in$ $K_{\infty}, \lambda \in(0,1)$ and the selections:

$$
\begin{aligned}
& \tilde{\gamma}_{1,2}(s):=\frac{s}{1-\sigma}, \tilde{\gamma}_{1,3}(s):=0 \\
& \tilde{\gamma}_{2,1}(s):=\lambda(1-\sigma) s, \tilde{\gamma}_{2,3}(s):=\gamma(s) \\
& \tilde{\gamma}_{3,1}(s):=\gamma^{-1}(\lambda(1-\sigma) s), \tilde{\gamma}_{3,2}(s):=\gamma^{-1}(\lambda s) .
\end{aligned}
$$

Since $\gamma \in K_{\infty} \cap C^{1}\left(\Re_{+}\right)$with $d \gamma / d s(0)>0$ there exist constants such that

$$
\gamma_{1} s \leq \gamma(s) \leq \gamma_{2} s, \quad \forall s \in[0, r]
$$

for $r>0$ sufficiently small. We notice that there exist $p, r>0$ such that the vector $k=(0,0,-p) \in \Re^{3}$ achieves $-x_{2}^{2}-p x_{2} x_{3} g(x) \leq-\sigma x_{2}^{2}$ for all $x \in \Re^{3}$ with $|x| \leq r$, $\max _{s=1,3} \tilde{\gamma}_{2, s}\left(\left|x_{s}\right|\right) \leq\left|x_{2}\right|$ and $x_{3} x_{1}^{2}-p x_{3}^{2} \leq-\sigma x_{3}^{2}$ for all $x \in \Re^{3}$ with $|x| \leq r, \max _{s=1,2} \tilde{\gamma}_{3, s}\left(\left|x_{s}\right|\right) \leq\left|x_{3}\right|$. More specifically, the constant $p>0$ must satisfy

$$
\frac{\lambda^{-1}(1-\sigma)^{-1} r \gamma_{2}+\sigma<p<\gamma_{1}(1-\sigma)}{(L r+|g(0)|)}
$$

where $L \geq 0$ is the Lipschitz constant that satisfies $|g(x)-g(0)| \leq L|x|$, for all $x \in \Re^{3}$ with $|x| \leq r$. Since $d \gamma / d s(0)>\sigma|g(0)| / 1-\sigma$, it follows from (36) that a selection of $p>0$ according to (37) is possible provided that $r>0$ is selected to be sufficiently small. Finally, we check implication (24). Indeed, using definitions (33), (34), (35), we conclude that implication (24) is equivalent to implication (32). The reader should notice that it might be possible to obtain a single CLF for system (30) of the form $V(x)=\varphi_{1}\left(x_{1}\right)+\varphi_{2}\left(x_{2}\right)+\varphi_{3}\left(x_{3}\right)$, or $V(x)=\max _{i=1,2,3} \varphi_{i}\left(x_{i}\right)$, where $\varphi_{i} \in C^{1}\left(\Re ; \Re_{+}\right)(i=1,2,3)$ are positive definite, radially unbounded functions (using the methodologies for the construction of Lyapunov functions in [5], [10], [11], [21], [22]). However, it should be noted how easily condition (32) was obtained from Corollary 4.1.

However, it should be noted that the use of auxiliary functions $\eta, W$ can give less demanding conditions for the existence of a stabilizing feedback. The following example illustrates this point.

Example 4.4: Again, we study problem (Q) for system (30). Here, we assume that $g: \Re^{3} \rightarrow \Re$ is independent of $x_{3}$, i.e., $g(x)=g\left(x_{1}, x_{2}\right)$. In this example, we show that system (30) can be stabilized globally by smooth feedback provided that there exist constants $\lambda \in(0,1), R>0, \sigma \in(0,1)$, functions $\gamma \in K_{\infty} \cap C^{1}\left(\Re_{+}\right)$with $d \gamma / d s(0)>\sigma|g(0)| / 1-\sigma$, $q \in C^{0}\left(\Re ; \Re_{+}\right)$with $0<q(x) \leq \sigma$ for all $x \neq 0$, such that the locally Lipschitz function $g: \Re^{3} \rightarrow \Re$ satisfies the following implication:

$$
\begin{aligned}
& \lambda(1-\sigma)\left|x_{1}\right| \leq \gamma\left(\left|x_{3}\right|\right), \lambda\left|x_{2}\right| \leq \gamma\left(\left|x_{3}\right|\right) \leq\left|x_{2}\right|, \\
& x_{1}^{2}+x_{2}^{2} \leq R, ; x_{2} x_{3} g(x)<0 \\
& \Rightarrow x_{2}^{2}\left(1-q\left(x_{2}\right)\right)+x_{2} x_{1}^{2} g(x) \geq\left|x_{2} x_{3} g(x)\right| q\left(x_{3}\right) .
\end{aligned}
$$

Implication (38) is less demanding than implication (32) since the inequality $x_{2}^{2}\left(1-Q\left(x_{2}\right)\right)+x_{2} x_{1}^{2} g(x) \geq$ $\left|x_{2} x_{3} g(x)\right| Q\left(x_{3}\right)$ is assumed to hold only for points that belong to the set

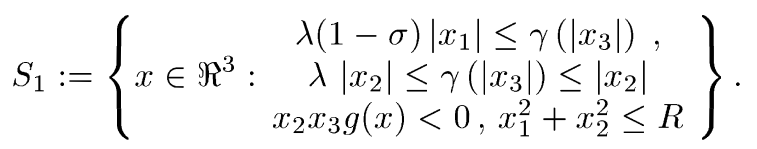

Notice that implication (32) requires that the inequality $x_{2}^{2}\left(1-Q\left(x_{2}\right)\right)+x_{2} x_{1}^{2} g(x) \geq\left|x_{2} x_{3} g(x)\right| Q\left(x_{3}\right)$ holds for points that belong to the set

$$
S_{2}:=\left\{x \in \Re^{3}: \begin{array}{c}
\lambda(1-\sigma)\left|x_{1}\right| \leq \gamma\left(\left|x_{3}\right|\right), x_{2} x_{3} g(x)<0 \\
\lambda\left|x_{2}\right| \leq \gamma\left(\left|x_{3}\right|\right) \leq\left|x_{2}\right|
\end{array}\right\}
$$

and $S_{1} \subseteq S_{2}$. For example, any function $g: \Re^{3} \rightarrow \Re$ with $g(x) x_{2} \geq 0$ for all $x \in \Re^{3}$ in the compact set

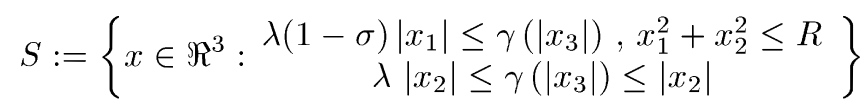

satisfies implication (38) for appropriate $q \in C^{0}\left(\Re ; \Re_{+}\right)$but does not necessarily satisfies implication (32).

In order to obtain condition (38), we apply Corollary 4.1 with $\eta(x):=-a+\left(x_{1}^{2}+x_{2}^{2}\right) / 2, W(x):=1+\left(x_{1}^{2}+x_{2}^{2}+x_{3}^{2}\right) / 2$, $\bar{k}(x) \equiv 0, \delta(\eta) \equiv c, K(\eta):=2(\eta+a)+c^{-1}, a \geq c>0$, $\varepsilon>0$ sufficiently small constants and an appropriate function $Q \in C^{0}\left(\Re ; \Re_{+}\right)$satisfying $0<Q(x) \leq \sigma$ for all $x \neq 0$, where $\sigma \in(0,1)$. By virtue of (38), we notice that implications (24), (25) and the small-gain conditions (6) hold for the selections given by (33), (34) and (35), provided that:

$$
2(a+\varepsilon) \leq R \text { and } Q(y) \leq q(y), \text { for all } y \in \Re .
$$

We notice that there exist $p, r>0$ such that the vector $k=$ $(0,0,-p) \in \Re^{3}$ achieves $-x_{2}^{2}-p x_{2} x_{3} g(x) \leq-\sigma x_{2}^{2}$ for all $x \in \Re^{3}$ with $|x| \leq r$ with $\max _{s=1,3} \tilde{\gamma}_{2, s}\left(\left|x_{s}\right|\right) \leq\left|x_{2}\right|$ and $x_{3} x_{1}^{2}-p x_{3}^{2} \leq-\sigma x_{3}^{2}$ for all $x \in \Re^{3}$ with $|x| \leq r$ with $\max _{s=1,2} \tilde{\gamma}_{3, s}\left(\left|x_{s}\right|\right) \leq\left|x_{3}\right|$. Furthermore, we obtain for all $x \in \Re^{3}$ satisfying $\eta(x) \geq 0$ :

$$
\begin{aligned}
& \sum_{i=1}^{3} \frac{\partial W}{\partial x_{i}}(x)\left(f_{i}(x)+g_{i}(x) \bar{k}(x)\right)=-x_{1}^{2}+x_{1} x_{2}-x_{2}^{2}+x_{3} x_{1}^{2} \\
& \leq x_{3} x_{1}^{2} \leq 2(\eta(x)+a) W(x) \leq K(\eta(x)) W(x) \\
& \sum_{i=1}^{3} \frac{\partial \eta}{\partial x_{i}}(x)\left(f_{i}(x)+g_{i}(x) \bar{k}(x)\right)=-x_{1}^{2}+x_{1} x_{2}-x_{2}^{2} \\
& \leq-\frac{\left(x_{1}^{2}+x_{2}^{2}\right)}{2}=-(\eta(x)+a) \leq-a \leq-c=-\delta(\eta(x))
\end{aligned}
$$

Finally, we check implications (26), (27). Implications (26) are equivalent to the implication:

$$
\begin{aligned}
& \text { "If } \lambda(1-\sigma)\left|x_{1}\right| \leq \gamma\left(\left|x_{3}\right|\right), \lambda\left|x_{2}\right| \leq \gamma\left(\left|x_{3}\right|\right), \\
& \begin{array}{l}
2 a \leq x_{1}^{2}+x_{2}^{2} \leq 2(a+\varepsilon) \text { and } x_{3} x_{2} g(x)<0 \\
\text { then }-x_{1}^{2}+x_{1} x_{2}-x_{2}^{2}-x_{2} g(x)\left(x_{1}^{2}+x_{3} Q\left(x_{3}\right)\right) \\
\quad \leq-\delta(\eta(x)) \text { " }
\end{array}
\end{aligned}
$$


and implications (27) are equivalent to the following:

$$
\begin{aligned}
& \text { "If } \lambda(1-\sigma)\left|x_{1}\right| \leq\left|x_{2}\right| \\
& \gamma\left(\left|x_{3}\right|\right) \leq\left|x_{2}\right|, 2 a \leq x_{1}^{2}+x_{2}^{2} \leq 2(a+\varepsilon) \\
& \text { and } x_{2} g(x)\left(x_{2} g(x)+x_{3}\right)<0 \text { then } \\
& -x_{1}^{2}+x_{1} x_{2}-x_{2}^{2}+x_{3} x_{1}^{2} \leq \\
& K(\eta(x)) W(x)+\frac{x_{2}\left(x_{2} g(x)+x_{3}\right)\left(Q\left(x_{2}\right)-1\right)}{g(x)} \\
& \text { "If } \lambda(1-\sigma)\left|x_{1}\right| \leq \gamma\left(\left|x_{3}\right|\right), \lambda\left|x_{2}\right| \leq \gamma\left(\left|x_{3}\right|\right) \\
& 2 a \leq x_{1}^{2}+x_{2}^{2} \leq 2(a+\varepsilon) \text { and } x_{3}\left(x_{2} g(x)+x_{3}\right)<0 \text { then } \\
& -x_{1}^{2}+x_{1} x_{2}-x_{2}^{2}-x_{2} g(x) x_{1}^{2} \leq \\
& K(\eta(x)) W(x)+\left(x_{2} g(x)+x_{3}\right) x_{3} Q\left(x_{3}\right) \text { ". }
\end{aligned}
$$

Since $g: \Re^{3} \rightarrow \Re$ is independent of $x_{3}$, it follows from (39), (42)-(44) that if $a, \varepsilon, c>0$ are sufficiently small then all requirements of Corollary 4.1 hold with $\delta(\eta) \equiv c, K(\eta):=$ $2(\eta+a)+c^{-1}$ and appropriate $Q \in C^{0}\left(\Re ; \Re_{+}\right)$.

\section{Stabilization of REACtion Networks}

Reaction networks taking place in Continuous Stirred Tank Reactors are described by ordinary differential equations of the form

$$
\dot{c}(t)=D(t)\left(c_{f}-c(t)\right)+S v(c(t))
$$

where $c(t) \in \operatorname{int}\left(\Re_{+}^{n}\right)$ is the vector of concentrations of all $n$ species, $v: \Re_{+}^{n} \rightarrow \Re_{+}^{m}$ is the continuously differentiable vector field of the $m$ reaction rates, $S \in \Re^{n \times m}$ is the stoichiometric matrix, $c_{f} \in \Re_{+}^{n}$ is the constant vector of the concentrations of the species at the reactor inlet (feed) and $D(t) \in\left[0, D_{\max }\right]$, where $D_{\max }>0$, is the dilution rate and is the ratio of the volumetric feed rate over the volume of the tank reactor. The dilution rate is used as input in many cases for the achievement of certain control objectives. The vector field $v(c)$ satisfies the condition

$$
\text { "If } c_{i}=0 \text { and } S_{i, j}<0 \text {, then } v_{j}(c)=0 "
$$

which expresses the (logical) requirement that a reaction cannot occur if one of its reactants is absent. The equilibrium points of (45) for $D=D^{*} \in\left(0, D_{\max }\right)$ satisfy the following equation:

$$
c_{f}=c^{*}-\left(D^{*}\right)^{-1} S v\left(c^{*}\right) .
$$

Notice that without loss of generality we may assume that $D^{*}=1 \in\left(0, D_{\max }\right)$ (time scaling). This section is devoted to the global stabilization problem of one of the equilibrium points of the reactor. More specifically, we study the reaction network (45) under the following assumptions:

(R1) There exist $N$ pairs of vectors $q_{l} \in \Re_{+}^{m}, p_{l} \in \Re^{n}(l=$ $1, \ldots, N)$ such that:

$$
S^{\prime} p_{l}=q_{l} \quad l=1, \ldots, N
$$

(R2) There exist constants $b, R>0$ such that

$\max _{i=1, \ldots, n}\left(c_{i}\right) \leq b+R \sum_{l=1}^{N} \max \left(p_{l}^{\prime} c_{f}-p_{l}^{\prime} c, 0\right)$, for all $c \in \Re_{+}^{n}$.

(R3) There exists $g \in K_{\infty}$ such that for all $j \in\{1, \ldots, m\}$, $i \in\{1, \ldots, n\}$ with $S_{i, j}<0$ it holds that

$$
0 \leq v_{j}(c) \leq g\left(\max _{j=1, \ldots, n}\left(c_{j}\right)\right) c_{i}, \text { for all } c \in \Re_{+}^{n} .
$$

The reader should notice that assumption (R3) is a consequence of (46) and the fact that $v(c)$ is a $C^{1}$ vector field. Assumptions (R1) and (R2) usually hold for reaction networks. Indeed, the total mass conservation requires the existence $p \in \Re^{n}$ such that $S^{\prime} p=0$, provided that all species are accounted in the model. Assumptions (R1) and (R2) can allow the case where some of the species are not accounted (because they are inert). Chemostat models (see [28]) satisfy assumptions (R1), (R2), and (R3).

The control problem of the global stabilization of one of the equilibrium points of (45) is a meaningful problem because many times there are multiple equilibrium points, indicating absence of global stability. The following example illustrates that multiple equilibrium points can occur even for simple reaction networks.

Example 5.1: Consider the simple reaction network $1 \rightarrow 2$ taking place in a CSTR

$$
\dot{c}(t)=D(t)\left(c_{f}-c(t)\right)+(-1,1)^{\prime} k c_{1}(t) c_{2}^{2}(t)
$$

where $c(t)=\left(c_{1}(t), c_{2}(t)\right)^{\prime} \in \operatorname{int}\left(\Re_{+}^{2}\right), k>0$ is a constant and $c_{f}=\left(c_{1, f}, c_{2, f}\right)^{\prime} \in \Re_{+}^{2}$ with $c_{1, f}>0$. The equilibrium points of (51) satisfy the equations

$$
c_{1, f}=c_{1}^{*}+k c_{1}^{*}\left(c_{2}^{*}\right)^{2}, \quad c_{2, f}=c_{2}^{*}-k c_{1}^{*}\left(c_{2}^{*}\right)^{2} .
$$

The above system of equations has a unique solution if $k\left(c_{1, f}+c_{2, f}\right)<3$. On the other hand, if $k\left(c_{1, f}+c_{2, f}\right)>3$ then (52) admits three different solutions. It is clear that the global stabilization problem for one of the equilibrium points of (51) is particularly meaningful for the case $k\left(c_{1, f}+c_{2, f}\right)>3$. The reader should notice that assumption (R1) holds with $p_{1}=(-1,-1)^{\prime}$ and $q_{1}=[0] \in \Re$. Inequality (49) with $R=1$ and $b:=c_{1, f}+c_{2, f}$ is a consequence of the inequality for all $c \in \Re_{+}^{2}$ :

$$
\max _{i=1,2}\left(c_{i}\right) \leq c_{1}+c_{2} \leq c_{1, f}+c_{2, f}+\max \left(p_{1}^{\prime} c_{f}-p_{1}^{\prime} c, 0\right) .
$$

Finally, assumption (R3) holds with $g(s):=k s^{2}$.

Without loss of generality, if there exists an equilibrium point $c^{*} \in \operatorname{int}\left(\Re_{+}^{n}\right)$ satisfying (47) with $D^{*}=1 \in\left(0, D_{\max }\right)$ then we may assume that $c^{*}=\mathbf{1}_{n}$. The following theorem provides sufficient conditions for the existence of a smooth stabilizing feedback. Its proof is given in the Appendix. 
Theorem 5.2: Consider system (45) under (R1), (R2), and (R3). Assume that $c^{*}=\mathbf{1}_{n} \in \operatorname{int}\left(\Re_{+}^{n}\right)$ satisfies (47) with $D^{*}=$ 1. Moreover, suppose that there exist $\tilde{\gamma}_{i, j} \in \mathcal{N}_{1}, i, j=1, \ldots, n$, with $\tilde{\gamma}_{i, i}(s) \equiv 0$ for $i=1, \ldots, n$ which satisfy the small-gain conditions (6), $\tilde{Q} \in C^{0}\left((0,+\infty) ; \Re_{+}\right)$with $\tilde{Q}(x)>0$ for all $x \in(0,1) \cup(1,+\infty)$ and constants $\varepsilon, \omega>0$ such that the following implications hold for $i, j \in\{1, \ldots, n\}$ :

$$
\begin{aligned}
& \text { "If } \max _{s=1, \ldots, n} \tilde{\gamma}_{i, s}\left(\left|\ln \left(c_{s}\right)\right|\right) \leq\left|\ln \left(c_{i}\right)\right|, \\
& \max _{s=1, \ldots, n} \tilde{\gamma}_{j, s}\left(\left|\ln \left(c_{s}\right)\right|\right) \leq\left|\ln \left(c_{j}\right)\right|, \\
& \sum_{l=1}^{N}\left(\max \left(p_{l}^{\prime} c_{f}-p_{l}^{\prime} c, 0\right)\right)^{2} \leq 2 \varepsilon \text { and one of the }
\end{aligned}
$$

statements (D1), (D2), (D3) and (D4) holds, then

$$
\begin{aligned}
& \frac{\left(c_{j, f}-c_{j}\right) \sum_{l=1}^{m} S_{i, l} v(c)-\left(c_{i, f}-c_{i}\right) \sum_{l=1}^{m} S_{j, l} v(c)}{c_{i} \ln \left(c_{i}\right) \tilde{Q}\left(c_{i}\right)\left(c_{j, f}-c_{j}\right)-c_{j} \ln \left(c_{j}\right) \tilde{Q}\left(c_{j}\right)\left(c_{i, f}-c_{i}\right)} \\
& \leq-1 . " \\
& \text { "If } c_{i}=c_{i, f}>0, \sum_{l=1}^{N}\left(\max \left(p_{l}^{\prime} c_{f}-p_{l}^{\prime} c, 0\right)\right)^{2} \leq 2 \varepsilon \text { and } \\
& \max _{s=1, \ldots, n} \tilde{\gamma}_{i, s}\left(\left|\ln \left(c_{s}\right)\right|\right) \leq\left|\ln \left(c_{i}\right)\right|, \\
& \text { then } \ln \left(c_{i, f}\right) \sum_{l=1}^{m} S_{i, l} v_{l}(c)+c_{i, f}\left(\ln \left(c_{i, f}\right)\right)^{2} \tilde{Q}\left(c_{i, f}\right) \leq 0 . "
\end{aligned}
$$

$$
\begin{aligned}
& \text { "If } \max _{s=1, \ldots, n} \tilde{\gamma}_{i, s}\left(\left|\ln \left(c_{s}\right)\right|\right) \leq\left|\ln \left(c_{i}\right)\right|, \\
& \varepsilon \leq \sum_{l=1}^{N}\left(\max \left(p_{l}^{\prime} c_{f}-p_{l}^{\prime} c, 0\right)\right)^{2} \leq 2 \varepsilon \text { and } \\
& \min \left(c_{i, f}, 1\right)<c_{i}<\max \left(c_{i, f}, 1\right), \\
& \text { then } \omega \leq-\frac{2 \varepsilon}{c_{i, f}-c_{i}}\left(c_{i} \ln \left(c_{i}\right) \tilde{Q}\left(c_{i}\right)+\sum_{j=1}^{m} S_{i, j} v_{j}(c)\right) .
\end{aligned}
$$

$$
\begin{aligned}
& \text { "If } \max _{s=1, \ldots, n} \tilde{\gamma}_{i, s}\left(\left|\ln \left(c_{s}\right)\right|\right) \leq\left|\ln \left(c_{i}\right)\right|, \\
& \sum_{l=1}^{N}\left(\max \left(p_{l}^{\prime} c_{f}-p_{l}^{\prime} c, 0\right)\right)^{2} \leq 2 \varepsilon \text { and } \\
& \min \left(c_{i, f}, 1\right)<c_{i}<\max \left(c_{i, f}, 1\right), \\
& \text { then } \ln \left(c_{i}\right) \sum_{l=1}^{m} S_{i, l} v_{l}(c)+c_{i}\left(\ln \left(c_{i}\right)\right)^{2} \tilde{Q}\left(c_{i}\right)<0 . " \\
& \text { "If } \max _{s=1, \ldots, n} \tilde{\gamma}_{i, s}\left(\left|\ln \left(c_{s}\right)\right|\right) \leq\left|\ln \left(c_{i}\right)\right|, \\
& \sum_{l=1}^{N}\left(\max \left(p_{l}^{\prime} c_{f}-p_{l}^{\prime} c, 0\right)\right)^{2} \leq 2 \varepsilon
\end{aligned}
$$$$
\text { and } c_{i}>\max \left(c_{i, f}, 1\right) \text { or } \min \left(c_{i, f}, 1\right)>c_{i} \text {, then }
$$$$
\ln \left(c_{i}\right) \sum_{l=1}^{m} S_{i, l} v_{l}(c)+c_{i}\left(\ln \left(c_{i}\right)\right)^{2} \tilde{Q}\left(c_{i}\right)
$$$$
+D_{\max }\left(c_{i, f}-c_{i}\right) \ln \left(c_{i}\right)<0 . "
$$

where the logical statements (D1), (D2), (D3), and (D4) are expressed by

(D1) $\min \left(c_{i, f}, 1\right)<c_{i}<\max \left(c_{i, f}, 1\right)$ and $c_{j}>\max \left(c_{j, f}, 1\right)$

(D2) $\min \left(c_{i, f}, 1\right)<c_{i}<\max \left(c_{i, f}, 1\right)$ and $\min \left(c_{j, f}, 1\right)>c_{j}$
(D3) $c_{i}>\max \left(c_{i, f}, 1\right)$ and $\min \left(c_{j, f}, 1\right)<c_{j}<\max \left(c_{j, f}, 1\right)$ (D4) $\min \left(c_{i, f}, 1\right)>c_{i}$ and $\min \left(c_{j, f}, 1\right)<c_{j}<\max \left(c_{j . f}, 1\right)$. Moreover, suppose that there exists $k=\left(k_{1}, \ldots, k_{n}\right)^{\prime} \in \Re^{n}$ such that

$$
\begin{array}{r}
\left(1+\sum_{j=1}^{n} k_{j} \ln \left(c_{j}\right)\right) \ln \left(c_{i}\right)\left(c_{i, f}-c_{i}\right) \\
+\ln \left(c_{i}\right) \sum_{j=1}^{m} S_{i, j} v_{j}(c) \\
\leq-\left(\ln \left(c_{i}\right)\right)^{2} c_{i} \tilde{Q}\left(c_{i}\right)
\end{array}
$$

for all $c \in \operatorname{int}\left(\Re_{+}^{n}\right)$ in a neighborhood of $\mathbf{1}_{n}$ with $\max _{s=1, \ldots, n} \tilde{\gamma}_{i, s}\left(\left|\ln \left(c_{s}\right)\right|\right) \leq\left|\ln \left(c_{i}\right)\right|(i=1, \ldots, n)$. Then there exists $\tilde{k} \in C^{\infty}\left(\operatorname{int}\left(\Re_{+}^{n}\right) ;\left[0, D_{\max }\right]\right)$, with $\tilde{k}\left(\mathbf{1}_{n}\right)=1$, such that $\mathbf{1}_{n} \in \operatorname{int}\left(\Re_{+}^{n}\right)$ is GAS for system (45) with $D=\tilde{k}(c)$.

The proof of Theorem 5.2 is based on Corollary 4.2 and the following methodology:

- First, we exploit the "mass balance" assumption (R1) in order to show that the solution of (45) necessarily enters to a certain region of the state space. This is accomplished by constructing appropriate functions $\eta, W, K$ which satisfy the properties assumed in Corollary 4.2. The solution of (45) remains in a compact subset of int $\left(\Re_{+}^{n}\right)$ during the transient period needed in order to enter the aforementioned region of the state space: this is guaranteed by assumptions (R2) and (R3).

- After the transient period, we utilize the vector Lyapunov function $V_{i}(c)=\left(\ln \left(c_{i}\right)\right)^{2} / 2, i=1, \ldots, n, i=1, \ldots, n$ and show that the assumptions of Corollary 4.2 hold.

The following example illustrates how Theorem 5.2 can be applied to reaction networks. Theorem 5.2 guarantees the existence of a globally stabilizing bounded feedback.

Example 5.1(continued): We turn to the global stabilization problem of one of the equilibrium points $c^{*} \in \operatorname{int}\left(\Re_{+}^{2}\right)$ of system (51) by means of smooth bounded feedback. In order to apply Theorem 5.2 we first apply a coordinate change that "brings" the equilibrium point to $1_{2} \in \operatorname{int}\left(\Re_{+}^{2}\right)$. Then system (51) takes the form

$$
\begin{aligned}
& \dot{c}(t)=D(t)\left(\left[\begin{array}{c}
1+\theta \\
1-\mu \theta
\end{array}\right]-c(t)\right)+\left[\begin{array}{c}
-1 \\
\mu
\end{array}\right] \theta c_{1}(t) c_{2}^{2}(t) \\
& c(t)=\left(c_{1}(t), c_{2}(t)\right)^{\prime} \in \operatorname{int}\left(\Re_{+}^{2}\right), D(t) \in\left[0, D_{\max }\right]
\end{aligned}
$$

where $\theta>0$ and $\mu \in\left(0, \theta^{-1}\right]$ are constant parameters $\left(k\left(c_{2}^{*}\right)^{2}=\theta, \quad \mu=c_{1}^{*} / c_{2}^{*}\right)$. For system (59), $\mathbf{1}_{2} \in \operatorname{int}\left(\Re_{+}^{2}\right)$ is the equilibrium point to be globally stabilized. Assumptions (R1)-(R3) hold with $N=1, q_{1}=[0] \in \Re_{+}^{1}$, $p_{1}=(-\mu,-1)^{\prime} \in \Re^{2}$. We further assume that $\mu<\theta^{-1}$, i.e., $c_{2, f}>0$ for (51). The problem that we study here is:

"How large must $D_{\max }>0$ be so that $\mathbf{1}_{2} \in \operatorname{int}\left(\Re_{+}^{2}\right)$ can be globally stabilized by a smooth feedback law $D=k(c)$ with $k\left(\mathbf{1}_{2}\right)=1$ and $k(c) \in\left[0, D_{\max }\right]$ for all $c \in \operatorname{int}\left(\Re_{+}^{2}\right)$ ?"”

We next show that all conditions of Theorem 5.2 hold provided that

$$
D_{\max }>(1+\mu)^{2} \text { and } D_{\max }>\frac{(1+\mu)\left(\theta(1+\mu)^{2}+1\right)}{\mu \theta} .
$$

We first check conditions Equations (54)- (58) of Theorem 5.2. Let $\varepsilon>0$ be an arbitrary constant, $\gamma \in K_{\infty}$ be an arbitrary function and select $\tilde{\gamma}_{1,2}(s):=\gamma(s), \tilde{\gamma}_{2,1}(s):=\gamma^{-1}(\lambda s)$ where 
$\lambda \in(0,1)$ is to be selected (notice that the small-gain conditions are automatically satisfied). Implication (54) holds provided that the inequality

$$
\begin{array}{r}
c_{1}\left(\ln \left(c_{1}\right)\right)^{2} \tilde{Q}\left(c_{1}\right)-c_{2}\left(\ln \left(c_{2}\right)\right)^{2} \tilde{Q}\left(c_{2}\right) \frac{\ln \left(c_{1}\right)\left(1+\theta-c_{1}\right)}{\ln \left(c_{2}\right)\left(1-\mu \theta-c_{2}\right)} \\
\leq \theta c_{1} c_{2}^{2} \ln \left(c_{1}\right) \frac{\mu+1-\mu c_{1}-c_{2}}{1-\mu \theta-c_{2}}
\end{array}
$$

holds for all $c \in \operatorname{int}\left(\Re_{+}^{2}\right)$ with $\gamma\left(\left|\ln \left(c_{2}\right)\right|\right) \leq\left|\ln \left(c_{1}\right)\right|$, $\lambda\left|\ln \left(c_{1}\right)\right| \leq \gamma\left(\left|\ln \left(c_{2}\right)\right|\right), \mu c_{1}+c_{2} \leq 1+\mu+\sqrt{2 \varepsilon}$ which satisfy one of the logical statements (D1), (D2), (D3) and (D4). It is clear that the previous inequality holds provided that the inequalities $\left(\ln \left(c_{1}\right)\right)^{2} \tilde{Q}\left(c_{1}\right) \leq \theta / 2 c_{2}^{2} \ln \left(c_{1}\right)$ $\mu+1-\mu c_{1}-c_{2} / 1-\mu \theta-c_{2}$ and $\left(\ln \left(c_{2}\right)\right)^{2} \tilde{Q}\left(c_{2}\right) \leq$ $\theta / 2 c_{1} c_{2} \ln \left(c_{2}\right) \mu c_{1}+c_{2}-\mu-1 / 1+\theta-c_{1}$ hold for all $c \in \operatorname{int}\left(\Re_{+}^{2}\right)$ with $\gamma\left(\left|\ln \left(c_{2}\right)\right|\right) \leq\left|\ln \left(c_{1}\right)\right|, \lambda\left|\ln \left(c_{1}\right)\right| \leq$ $\gamma\left(\left|\ln \left(c_{2}\right)\right|\right), \mu c_{1}+c_{2} \leq 1+\mu+\sqrt{2 \varepsilon}$ which satisfy one of the logical statements (D1), (D2), (D3), and (D4). The above requirements imply that:

- If (D1) holds $1<c_{1}<1+\theta, 1<c_{2} \leq$ $\exp \left(\gamma^{-1}\left(\ln \left(c_{1}\right)\right)\right)$, then we must have $\tilde{Q}\left(c_{1}\right) \leq$ $c_{1}-1 / 2 \ln \left(c_{1}\right)$ and $\tilde{Q}\left(c_{2}\right) \leq c_{2}-1 / 2 \ln \left(c_{2}\right) c_{2}$.

- If (D2) holds, i.e., $1<c_{1}<1+\theta$ and $\exp \left(-\gamma^{-1}\left(\ln \left(c_{1}\right)\right)\right) \leq c_{2}<1-\mu \theta$, then we must have $\tilde{Q}\left(c_{1}\right) \leq \theta / 2 \ln \left(c_{1}\right) \exp \left(-2 \gamma^{-1}\left(\ln \left(c_{1}\right)\right)\right)$ and $\tilde{Q}\left(c_{2}\right) \leq 1-c_{2} / 2\left|\ln \left(c_{2}\right)\right| c_{2}$.

- If (D3) holds $c_{1}>1+\theta, 1-\mu \theta<c_{2}<1$, then we must have $\tilde{Q}\left(c_{1}\right) \leq c_{1}-1 / 2 \ln \left(c_{1}\right)(1-\mu \theta)^{2}$ and $\tilde{Q}\left(c_{2}\right) \leq$ $(1+\theta) 1-c_{2} / 2\left|\ln \left(c_{2}\right)\right| c_{2}$

- If (D4) holds, i.e., $1-\mu \theta<c_{2}<1$ and $\exp \left(-\lambda^{-1} \gamma\left(\left|\ln \left(c_{2}\right)\right|\right)\right) \leq c_{1}<1$, then we must have $\tilde{Q}\left(c_{1}\right) \leq 1-c_{1} / 2\left|\ln \left(c_{1}\right)\right|(1-\mu \theta)^{2}$ and $\tilde{Q}\left(c_{2}\right) \leq 1-c_{2} / 2\left|\ln \left(c_{2}\right)\right| c_{2} \exp \left(-\lambda^{-1} \gamma\left(\left|\ln \left(c_{2}\right)\right|\right)\right)$.

Consequently, implication (54) automatically holds, if $\tilde{Q} \in$ $C^{0}\left((0,+\infty) ; \Re_{+}\right)$is selected to be

$$
\begin{aligned}
\tilde{Q}(1) & :=\frac{A(1-\mu \theta)^{2}}{2} \\
\tilde{Q}(c) & :=A \frac{\min (1,|1-c|)}{2|\ln (c)|} \min \left(c,(1-\mu \theta)^{2}\right) \Xi(c) \\
\Xi(c) & :=\exp \left(-\max \left(\lambda^{-1} \gamma(|\ln (c)|), 2 \gamma^{-1}(|\ln (c)|)\right)\right), \\
\text { for } c & \in(0,1) \cup(1,+\infty)
\end{aligned}
$$

where $A \in(0,1]$ is a constant (yet to be selected). Conditions (55) with $i=1,2$ give the inequalities $\tilde{Q}(1+\theta) \leq$ $\theta / \ln (1+\theta) \exp \left(-2 \gamma^{-1}(\ln (1+\theta))\right)$ and $\tilde{Q}(1-\mu \theta) \leq$ $\mu \theta(1-\mu \theta) /|\ln (1-\mu \theta)| \exp \left(-\lambda^{-1} \gamma(|\ln (1-\mu \theta)|)\right)$, which hold automatically for $\tilde{Q}$ defined by (61), (62). Conditions (56) with $i=1,2$ and $\omega>0$ hold provided that

$$
\begin{aligned}
& A \leq \frac{1}{2(1+\theta)} \exp \left(-2 \gamma^{-1}(\ln (1+\theta))\right) \\
& \omega \leq \frac{\varepsilon \theta}{1+\theta} \exp \left(-2 \gamma^{-1}(\ln (1+\theta))\right) \\
& \text { and } A \leq \exp \left(-\lambda^{-1} \gamma(|\ln (1-\mu \theta)|)\right) \\
& \omega \leq \varepsilon(1-\mu \theta)^{2} \exp \left(-\lambda^{-1} \gamma(|\ln (1-\mu \theta)|)\right)
\end{aligned}
$$

and conditions (57) hold automatically for the above selections. Finally, we check condition (58). Conditions (58) hold provided that $D_{\max }>c_{2}^{2}$ and $D_{\max }>\mu \theta c_{1} c_{2}^{2}+c_{2} \ln \left(c_{2}\right) \tilde{Q}\left(c_{2}\right) / \mu \theta$ for all $c \in \operatorname{int}\left(\Re_{+}^{2}\right)$ with $\mu c_{1}+c_{2} \leq(1+\mu)+\sqrt{2 \varepsilon}$. Since $\varepsilon>0$ is arbitrary, we conclude (by virtue of (60)) that the above inequalities hold.

The existence of a vector $k=\left(k_{1}, k_{2}\right)^{\prime} \in \Re^{2}$ with

$$
\begin{array}{r}
\left(1+\sum_{j=1}^{2} k_{j} \ln \left(c_{j}\right)\right) \ln \left(c_{i}\right)\left(c_{i, f}-c_{i}\right) \\
+\ln \left(c_{i}\right) \sum_{j=1}^{m} S_{i, j} v_{j}(c) \\
\leq-\left(\ln \left(c_{i}\right)\right)^{2} c_{i} \tilde{Q}\left(c_{i}\right)
\end{array}
$$

for all $c$ in a neighborhood of $\mathbf{1}_{2}$ with $\max _{s=1, \ldots, n} \tilde{\gamma}_{i, s}\left(\left|\ln \left(c_{s}\right)\right|\right) \leq$ $\left|\ln \left(c_{i}\right)\right|(i=1,2)$ is guaranteed by the observation that the unbounded smooth feedback law $D=c_{2}^{2}$ guarantees the inequalities $D \ln \left(c_{i}\right)\left(c_{i, f}-c_{i}\right)+\ln \left(c_{i}\right) \sum_{j=1}^{m} S_{i, j} v_{j}(c) \leq$ $-\left(\ln \left(c_{i}\right)\right)^{2} c_{i} \tilde{Q}\left(c_{i}\right)$ for appropriate $\tilde{\gamma}_{1,2}, \tilde{\gamma}_{2,1} \in K_{\infty}$ and for all $c$ in a neighborhood of $\mathbf{1}_{2}$ with $\max _{s=1, \ldots, n} \tilde{\gamma}_{i, s}\left(\left|\ln \left(c_{s}\right)\right|\right) \leq$ $\left|\ln \left(c_{i}\right)\right|(i=1,2)$.

\section{CONCLUDING REMARKS}

This paper has shown how recent results on vector Lyapunov functions can be used for smooth globally stabilizing feedback design for nonlinear systems. In particular, Theorem 3.4 provides necessary and sufficient conditions based on vector control Lyapunov functions for the existence of a smooth global stabilizer for affine in the control uncertain nonlinear systems.

The flexibility of vector Lyapunov functions is a feature that can be exploited for feedback design in large scale systems. Corollaries 4.1 and 4.2 provide practically checkable sufficient conditions for the existence of a stabilizer for nonlinear systems. Corollaries 4.1 and 4.2 are direct applications of Theorem 3.4 and show how vector Lyapunov functions can lead to results which are not easily obtained by the classical single Lyapunov analysis. The obtained results are exploited for the derivation of sufficient conditions which guarantee stabilizability of the equilibrium point of a reaction network taking place in a continuous stirred tank reactor. Future research may include:

i) the extension of the present results to the multiple-input case,

ii) the development of explicit formulas for the feedback stabilizers which are designed based on VRCLFs,

iii) the development of "adding an integrator"-like results based on VRCLFs, which can allow important modifications to the backstepping methodology (see [17]).

It should be noted that the extension of the results to the multiple-input case is not straightforward. The reason is that a system of linear inequalities must be verified and if more than one inputs are present then one cannot use Lemma 3.5. However, other tools (Farkas' lemma) might be helpful.

\section{APPENDIX}

Proof of Lemma 3.5: Define $B^{0}:=$ $\left\{i \in\{1, \ldots, m\}: g_{i}=0\right\}, B^{+}:=\left\{i \in\{1, \ldots, m\}: g_{i}>0\right\}$ and $B^{-}:=\left\{i \in\{1, \ldots, m\}: g_{i}<0\right\}$. Notice that implication (I) gives:

$$
\text { "If } B^{0} \neq \emptyset \text {, then } \max _{i \in B^{0}}\left(f_{i}\right)<0 . "
$$


and implication (II) gives:

"If $B^{+} \neq \emptyset$ and $B^{-} \neq \emptyset$, then $\max _{i \in B^{+}}\left(\frac{f_{i}}{g_{i}}\right)<\min _{i \in B^{-}}\left(\frac{f_{i}}{g_{i}}\right)$ ".

Consider all possible cases:

(a) $B^{+}=\emptyset=B^{-}$. In this case (64) implies that we can select arbitrary $u \in \Re$ so that $f_{i}+g_{i} u<0$ for all $i=$ $1, \ldots, m$.

(b) $B^{+}=\emptyset \neq B^{-}$. In this case, we can select $u>\max _{i \in B^{-}}\left(-f_{i} / g_{i}\right)$. The previous inequality in conjunction with (64) implies that $f_{i}+g_{i} u<0$ for all $i=1, \ldots, m$.

(c) $B^{-}=\emptyset \neq B^{+}$. In this case, we can select $u<\min _{i \in B^{+}}\left(-f_{i} / g_{i}\right)$. The previous inequality in conjunction with (64) implies that $f_{i}+g_{i} u<0$ for all $i=1, \ldots, m$.

(d) $B^{+} \neq \emptyset \neq B^{-}$. In this case (65) implies $\max _{i \in B^{+}}\left(f_{i} / g_{i}\right)<$ $\min _{i \in B^{-}}\left(f_{i} / g_{i}\right)$. Therefore, the inequality $\min _{i \in B^{+}}\left(-f_{i} / g_{i}\right)=$ $-\max _{i \in B^{+}}\left(f_{i} / g_{i}\right)>-\min _{i \in B^{-}}\left(f_{i} / g_{i}\right)=\max _{i \in B^{-}}\left(-f_{i} / g_{i}\right)$ holds. In this case, we can select $u \in \Re$ so that $\min _{i \in B^{+}}\left(-f_{i} / g_{i}\right)>u>\max _{i \in B^{-}}\left(-f_{i} / g_{i}\right)$. The previous inequality in conjunction with (64) implies that $\max _{i=1, \ldots, m}\left(f_{i}+g_{i} u\right)<0$.

Notice that the above selections in each case guarantee that there exists $u \in(-a,+\infty)$ so that $f_{i}+g_{i} u<0$ for all $i=1, \ldots, m$, provided that either $B^{+}=\emptyset$ or $B^{+} \neq \emptyset$ and $\min _{i \in B^{+}}\left(-f_{i} / g_{i}\right)>-a$. Implication (III) guarantees that one of the previously mentioned cases holds. Finally, notice that the above selections in each case guarantee that there exists $u \in(-a, b)$ so that $f_{i}+g_{i} u<0$ for all $i=1 \ldots, m$, provided that the following implications hold: (i) if $B^{+} \neq \emptyset$ then $\min _{i \in B^{+}}\left(-f_{i} / g_{i}\right)>-a$, (ii) if $B^{-} \neq \emptyset$ then $\max _{i \in B^{-}}\left(-f_{i} / g_{i}\right)<b$. $i \in B^{+}$
Implications (III) and (IV) guarantee that the previous implications hold.

The converse statements are proved in the same way by distinguishing the above cases. The proof is complete. $\triangleleft$

Proof of Lemma 3.6: The methodology of the proof is to show that every $\gamma_{i, j} \in \mathcal{N}_{1}(i, j=1, \ldots, m, i \neq j)$ can be replaced by a function $\tilde{\gamma}_{i, j} \in \mathcal{N}_{1}$ which is positive definite and satisfies $\lim _{s \rightarrow+\infty} \tilde{\gamma}_{i, j}(s)=+\infty$ in such a way that the "new" set of functions $\gamma_{p, q} \in \mathcal{N}_{1}(p, q=1, \ldots, m)$ with $\gamma_{i, j} \in \mathcal{N}_{1}$ replaced by $\tilde{\gamma}_{i, j} \in \mathcal{N}_{1}$ satisfies all properties of the VRCLF.

A key observation is that all inequalities (9), (10), (14), (15), (16), (17) and (20) hold automatically if $\gamma_{i, j} \in \mathcal{N}_{1}$ is replaced by a function $\tilde{\gamma}_{i, j} \in \mathcal{N}_{1}$ satisfying:

$$
\gamma_{i, j}(s) \leq \tilde{\gamma}_{i, j}(s), \text { for all } s \geq 0 .
$$

The only thing that remains to be checked is the set of smallgain inequalities (6). The inequalities (6) which are affected by the replacement of $\gamma_{i, j} \in \mathcal{N}_{1}$ with the function $\tilde{\gamma}_{i, j} \in \mathcal{N}_{1}$ can be expressed by the following inequality:

$$
\tilde{\gamma}_{i, j}(a(s))<s, \text { for all } s>0
$$

where $a \in \mathcal{N}_{1}$ is defined as follows:

" $a(s)$ for a given $s \geq 0$ is the maximum of $\gamma_{j, i}(s)$ and the maximum of all $\left(\gamma_{i, z_{1}} \circ \gamma_{z_{1}, z_{2}} \circ \cdots \circ \gamma_{z_{l-1}, z_{l}} \circ \gamma_{z_{l}, j}\right)(s)$ over the set of all indices $\left\{z_{1}, \ldots, z_{l}\right\} \subseteq\{1, \ldots, m\} \backslash\{i, j\}$, with $z_{p} \neq z_{q}$ if $p \neq q$ "

To see this, notice that each inequality (6) which is affected by the replacement of $\gamma_{i, j} \in \mathcal{N}_{1}$ with the function $\tilde{\gamma}_{i, j} \in \mathcal{N}_{1}$ is guaranteed by (67) and the following fact:

$$
\begin{aligned}
& \text { "If } \gamma_{1}\left(\gamma_{2}(s)\right)<s \text { for all } s>0 \text { for a given pair of } \\
& \gamma_{1}, \gamma_{2} \in \mathcal{N}_{1} \text { then } \gamma_{2}\left(\gamma_{1}(s)\right)<s \text { for all } s>0 \text { ". }
\end{aligned}
$$

Notice that since inequalities (6) hold with the original $\gamma_{i, j} \in$ $\mathcal{N}_{1}$, it holds that:

$$
\gamma_{i, j}(a(s))<s, \text { for all } s>0
$$

Define next:

$$
\tilde{\gamma}_{i, j}(s):=\max \left(\gamma_{i, j}(s), \frac{1}{2} \tilde{a}^{-1}(s)\right), \text { for all } s \geq 0
$$

where $\tilde{a}(s):=a(s)+s$. Definition (69) and inequality (68) guarantee that inequalities (66) and (67) hold. Moreover, since $\tilde{a}^{-1} \in K_{\infty}$ it follows that $\tilde{\gamma}_{i, j} \in \mathcal{N}_{1}$ and satisfies $\lim _{\rightarrow+\infty} \tilde{\gamma}_{i, j}(s)=+\infty$. The proof is complete.

Proof of Theorem 3.4: Without loss of generality and since $\eta(0)<0$ we may assume that the neighborhood $A$ involved in property (vi) satisfies $A \subset\left\{x \in \Re^{n}: \eta(x)<0\right\}$. Let $r>0$ with $\left\{x \in \Re^{n}:|x| \leq 2 r\right\} \subset A$. Moreover, by virtue of Lemma 3.6, without loss of generality we may assume that all functions $\gamma_{i, j} \in \mathcal{N}_{1}, i, j=1, \ldots, m$, are positive definite for $i \neq j$. Using properties (ii), (iii), (iv), convexity of $U \subseteq \Re$ and partition of unity arguments, we next construct smooth feedback laws $k_{1}:\left\{x \in \Re^{n}: \eta(x)>0\right\} \rightarrow U$, $k_{2} \quad: \quad\left\{x \in \Re^{n}: \eta(x)<\varepsilon, x \neq 0\right\} \rightarrow U \quad$ and $k_{3}:\left\{x \in \Re^{n}: 0<\eta(x)<\varepsilon\right\} \rightarrow U$ such that the following hold:

$$
\begin{aligned}
& 2 H(x)+2 k_{1}(x) L_{g} \eta(x)-\delta(\eta(x)) \leq 0 \\
& \text { for all } x \in\left\{x \in \Re^{n}: \eta(x)>0\right\} \\
& R(x)+k_{1}(x) L_{g} W(x)-K(\eta(x)) W(x) \leq 0 \\
& \text { for all } x \in\left\{x \in \Re^{n}: \eta(x)>0\right\} \\
& \text { If } \max _{j=1, \ldots, m} \gamma_{i, j}\left(V_{j}(x)\right) \leq V_{i}(x), x \neq 0 \text { and } \eta(x)<\varepsilon \\
& \text { then } 2 F_{i}(x)+2 k_{2}(x) L_{g} V_{i}(x)-\rho\left(V_{i}(x)\right) \leq 0 \text {. } \\
& 2 H(x)+2 k_{3}(x) L_{g} \eta(x)-\delta(\eta(x)) \leq 0 \\
& \text { for all } x \in\left\{x \in \Re^{n}: 0<\eta(x)<\varepsilon\right\} \\
& R(x)+k_{3}(x) L_{g} W(x)-K(\eta(x)) W(x) \leq 0 \\
& \text { for all } x \in\left\{x \in \Re^{n}: 0<\eta(x)<\varepsilon\right\} \\
& \text { If } \max _{j=1, \ldots, m} \gamma_{i, j}\left(V_{j}(x)\right) \leq V_{i}(x), x \neq 0 \text { and } 0<\eta(x)<\varepsilon, \\
& \text { then } 2 F_{i}(x)+2 k_{3}(x) L_{g} V_{i}(x)-\rho\left(V_{i}(x)\right) \leq 0 .
\end{aligned}
$$

Let $p: \Re \longrightarrow[0,1]$ be a smooth non-decreasing function with $p(s)=0$ for all $s \leq 0$ and $p(s)=1$ for all $s \geq 1$. Define:

$\tilde{k}(x):=k_{1}(x)$ for all $x \in\left\{x \in \Re^{n}: 5 \eta(x)>4 \varepsilon\right\}$

$\tilde{k}(x):=k_{2}(x)$

for all $x \in\left\{x \in \Re^{n}: 5 \eta(x)<\varepsilon\right\} \cap\left\{x \in \Re^{n}:|x|>2 r\right\}$

$\tilde{k}(x):=k_{3}(x)$ for all $x \in\left\{x \in \Re^{n}: 2 \varepsilon<5 \eta(x)<3 \varepsilon\right\}$ 
$\tilde{k}(x):=\left(1-p\left(\frac{5 \eta(x)}{\varepsilon}-3\right)\right) k_{3}(x)+p\left(\frac{5 \eta(x)}{\varepsilon}-3\right) k_{1}(x)$

for all $x \in\left\{x \in \Re^{n}: 3 \varepsilon \leq 5 \eta(x) \leq 4 \varepsilon\right\}$

$\tilde{k}(x):=\left(1-p\left(\frac{5 \eta(x)}{\varepsilon}-1\right)\right) k_{2}(x)+p\left(\frac{5 \eta(x)}{\varepsilon}-1\right) k_{3}(x)$

for all $x \in\left\{x \in \Re^{n}: \varepsilon \leq 5 \eta(x) \leq 2 \varepsilon\right\}$

$\tilde{k}(x):=k(x)$ for all $x \in\left\{x \in \Re^{n}:|x|<r\right\}$

$\tilde{k}(x):=\left(1-p\left(\frac{|x|^{2}-r^{2}}{3 r^{2}}\right)\right) k(x)+p\left(\frac{|x|^{2}-r^{2}}{3 r^{2}}\right) k_{2}(x)$

for all $x \in\left\{x \in \Re^{n}: r \leq|x| \leq 2 r\right\}$

where $k \in C^{\nu}(A ; U)$ is the locally Lipschitz mapping involved in property (vi). It follows directly from assumption $(\mathrm{H})$, compactness of $D \subseteq \Re^{l}$ and the fact that the mapping $\tilde{k}: \Re^{n} \rightarrow U$ defined above is locally Lipschitz with $\tilde{k}(0)=0$, that the closed-loop system (7) with $u=\hat{k}(x)$ satisfies assumptions (A1-3) with $h(x):=\eta(x)-2 \varepsilon / 5, \rho_{i}(s):=\rho(s) / 2(i=1, \ldots, m)$ and $\tilde{\delta}(s):=\delta(s+2 \varepsilon / 5) / 2 \tilde{K}(s):=2 K(s+2 \varepsilon / 5)$ in place of $\delta \in C^{0}\left(\Re_{+} ;(0,+\infty)\right), K \in C^{0}\left(\Re_{+} ;[1,+\infty)\right)$. Consequently, we conclude from Theorem 2.7 in [15] that the closed-loop system (7) with is RGAS.

Therefore, we are left with the task of constructing smooth feedback laws $k_{1}, k_{2}$ and $k_{3}$ so that (70)-(75) hold.

Construction of $k_{1}:\left\{x \in \Re^{n}: \eta(x)>0\right\} \rightarrow U$. By virtue of (11), (12), (13), (18), (19), (21), (22), and Lemma 3.5, it follows that for every $x \in \Re^{n}$ with $\eta(x)>0$ there exists $u_{x} \in U$ with $4 H(x)+4 u_{x} L_{g} \eta(x)-\delta(\eta(x)) \leq 0$ and $2 R(x)+2 u_{x} L_{g} W(x) \leq K(\eta(x)) W(x)$. Continuity of $H(x), L_{g} \eta(x), \delta(\eta(x)), \quad R(x), L_{g} W(x), K(\eta(x)), W(x)$ implies that there exists $\delta_{x}>0$ such that $\eta(y)>0$, $2 H(y)+2 u_{x} L_{q} \eta(y)-\delta(\eta(y)) \leq 0, R(y)+u_{x} L_{g} W(y)-$ $K(\eta(y)) W(y) \leq 0$ for all $y \in \Re^{n}$ with $|y-x| \leq \delta_{x}$.

Therefore, the sets $\left\{y \in \Re^{n}:|y-x|<\delta_{x}\right\} \quad$ for all $x \in \Re^{n}$ with $\eta(x)>0$ form an open covering of the open set $\left\{x \in \Re^{n}: \eta(x)>0\right\}$. By partition of unity, there exists a family of smooth functions $\phi_{i}:\left\{x \in \Re^{n}: \eta(x)>0\right\} \rightarrow[0,1], i=1,2, \ldots$ such that:

a) For every $i=1,2, \ldots$ there exists $x_{i} \in \Re^{n}$ with $\eta\left(x_{i}\right)>$ 0 and $\operatorname{supp}\left(\phi_{i}\right) \subset\left\{y \in \Re^{n}:\left|y-x_{i}\right|<\delta_{x_{i}}\right\}$.

b) The sum $\sum_{i} \phi_{i}(x)$ is locally finite and satisfies $\sum_{i=1}^{\infty} \phi_{i}(x)=1$ for all $x \in \Re^{n}$ with $\eta(x)>0$.

We define for all $x \in \Re^{n}$ with $\eta(x)>0$ :

$$
k_{1}(x):=\sum_{i=1}^{\infty} u_{x_{i}} \phi_{i}(x)
$$

We first notice that (by local finiteness) the mapping $k_{1}$ defined by (83) is smooth and satisfies $k_{1}(x) \in U$ for all $x \in \Re^{n}$ with $\eta(x)>0$. For arbitrary $x \in \Re^{n}$ with $\eta(x)>0$ we define the finite set $J_{x} \subset\{1,2, \ldots\}$ of indices such that $\phi_{i}(x)>0$. It follows that $x \in\left\{y \in \Re^{n}:\left|y-x_{j}\right|<\delta_{x_{j}}\right\}$ for all $j \in$ $J_{x}$. Therefore, we get $2 H(x)+2 u_{x_{j}} L_{g} \eta(x)-\delta(\eta(x)) \leq 0$, $R(x)+u_{x_{j}} L_{g} W(x)-K(\eta(x)) W(x) \leq 0$ for all $j \in J_{x}$. Using the previous inequalities, the fact that $\sum_{j \in J_{x}} \phi_{j}(x)=1$ and definition (83) we obtain (70) and (71).

Construction of $k_{2}:\left\{x \in \Re^{n}: \eta(x)<\varepsilon, x \neq 0\right\} \rightarrow U$. Let $x \in \Re^{n}$ with $\eta(x)<\varepsilon, x \neq 0$ be arbitrary. Let $J^{+}(x)$ be the set of all $j \in\{1, \ldots, m\}$ such that $\max _{s=1, m} \gamma_{j, s}\left(V_{s}(x)\right) \leq V_{j}(x)$. Since all $\gamma_{i, j} \mathcal{N}_{1}, i, j=1, \ldots, m$ are positive definite, we have $V_{j}(x)>0$ for all $j \in J^{+}(x)$. By virtue of (9), (10), (17), (20) and Lemma 3.5, there exists $u_{x} \in U$ with

$$
4 F_{j}(x)+4 u_{x} L_{g} V_{j}(x)-\rho\left(V_{j}(x)\right) \leq 0 \text {, for all } j \in J^{+}(x) .
$$

Define $J^{-}(x)$ to be the set of all $j \in\{1, \ldots, k\}$ such that $\max _{s=1} \gamma_{j, s}\left(V_{s}(x)\right)>V_{j}(x)$. Notice that $J^{+}(x) \cup J^{-}(x)=$ $\{1, \ldots, m\}$ for all $x \in \Re^{n}$ with $\eta(x)<\varepsilon, x \neq 0$. Continuity of mappings $F_{j}(x), L_{g} V_{j}(x), \rho\left(V_{j}(x)\right)(j=1, \ldots, m)$ $\gamma_{i, j} \mathcal{N}_{1}, i, j=1, \ldots, m$ implies that there exists $\delta_{x}>0$ such that $\eta(y)<\varepsilon, y \neq 0, \max _{s=1, m_{m}} \gamma_{j, s}\left(V_{s}(y)\right)>V_{j}(y)$ for all $j \in J^{-}(x), 2 F_{j}(y)+2 u_{x} L_{g} V_{j}(y)-\rho\left(V_{j}(y)\right) \leq 0$ for all $j \in J^{+}(x)$ and for all $y \in \Re^{n}$ with $|y-x| \leq \delta_{x}$. Notice that the previous inequality imply that $J^{+}(y) \subseteq J^{+}(x)$ for all $y \in \Re^{n}$ with $|y-x| \leq \delta_{x}$. Therefore, we have

$$
\begin{aligned}
& 2 F_{j}(y)+2 u_{x} L_{g} V_{j}(y)-\rho\left(V_{j}(y)\right) \leq 0, \\
& \text { for all } j \in J^{+}(y) \text { and } y \in \Re^{n} \text { with }|y-x| \leq \delta_{x} .
\end{aligned}
$$

Therefore, the sets $\left\{y \in \Re^{n}:|y-x|<\delta_{x}\right\}$ for all $x \in \Re^{n}$ with $\eta(x)<\varepsilon, x \neq 0$ form an open covering of the open set $\left\{x \in \Re^{n}: \eta(x)<\varepsilon, x \neq 0\right\}$. By partition of unity, there exists a family of smooth functions $\phi_{i}:\left\{x \in \Re^{n}: \eta(x)<\varepsilon, x \neq 0\right\} \rightarrow[0,1], i=1,2, \ldots$ such that:

a) For every $i=1,2, \ldots$ there exists $x_{i} \in \Re^{n}$ with $\eta\left(x_{i}\right)<$ $\varepsilon, x_{i} \neq 0$ and $\operatorname{supp}\left(\phi_{i}\right) \subset\left\{y \in \Re^{n}:\left|y-x_{i}\right|<\delta_{x_{i}}\right\}$.

b) The sum $\sum_{i} \phi_{i}(x)$ is locally finite and satisfies $\sum_{i=1}^{\infty} \phi_{i}(x) \stackrel{\sum_{i}}{=} 1$ for all $x \in \Re^{n}$ with $\eta(x)<\varepsilon$, $x \neq 0$.

We define for all $x \in \Re^{n}$ with $\eta(x)<\varepsilon, x \neq 0$ :

$$
k_{2}(x):=\sum_{i=1}^{\infty} u_{x_{i}} \phi_{i}(x) .
$$

We first notice that (by local finiteness) the mapping $k_{2}$ defined by (86) is smooth and satisfies $k_{2}(x) \in U$ for all $x \in \Re^{n}$ with $\eta(x)<\varepsilon, x \neq 0$. For arbitrary $x \in \Re^{n}$ with $\eta(x)<\varepsilon, x \neq$ 0 we define the finite set $I_{x} \subset\{1,2, \ldots\}$ of indices such that $\phi_{i}(x)>0$. It follows that $x \in\left\{y \in \Re^{n}:\left|y-x_{i}\right|<\delta_{x_{i}}\right\}$ for all $i \in I_{x}$. Therefore, by virtue of (85), the fact that $\sum_{i \in I_{x}} \phi_{i}(x)=$ 1 , the fact that $J^{+}(x)$ is the set of all $j \in\{1, \ldots, m\}$ such that $\max _{s=1} \gamma_{j, s}\left(V_{s}(x)\right) \leq V_{j}(x)$ and definition (86), we obtain (72).

The construction of $k_{3}:\left\{x \in \Re^{n}: 0<\eta(x)<\varepsilon\right\} \rightarrow U$ is similar to the constructions of $k_{1}$ and $k_{2}$.

Proof of Theorem 5.2: The proof utilizes Corollary 4.2 for the system obtained by the following change of coordinates and the input transformation:

$$
c=\exp (x), \quad D=1+u
$$

namely, the system:

$$
\dot{x}_{i}(t)=(1+u(t))\left(c_{i, f} e^{-x_{i}(t)}-1\right)+e^{-x_{i}(t)} \sum_{j=1}^{m} S_{i, j} v_{j}\left(e^{x(t)}\right)
$$


for $i=1 \ldots n$. It follows from (87) that $u(t) \in U:=$ $\left[-1, D_{\max }-1\right]$ and consequently assumption (P3) holds for system (88). Moreover, $D_{\max }-1>0$. Define

$$
\begin{array}{r}
P(i):=\left\{j \in\{1, \ldots, m\}: S_{i, j}<0\right\} \\
\text { and } \sigma:=\max _{i=1, \ldots, n}\left(\sum_{j \in P(i)}\left|S_{i, j}\right|\right) .
\end{array}
$$

We apply Corollary 4.2 with $f_{i}(x)=c_{i, f} e^{-x_{i}}-1+$ $e^{-x_{i}} \sum_{j=1}^{m} S_{i, j} v_{j}\left(e^{x}\right), g_{i}(x)=c_{i, f} e^{-x_{i}}-1$ for $i=1, \ldots, n$ and

$$
\begin{aligned}
\eta(x) & :=\sum_{l=1}^{N}\left(\max \left(p_{l}^{\prime} c_{f}-p_{l}^{\prime} e^{x}, 0\right)\right)^{2}-\varepsilon \\
W(x) & :=1+\varepsilon+\eta(x)+\sum_{i=1}^{n} e^{-x_{i}} \\
\bar{k}(x) & \equiv 0, \delta(\eta) \equiv \min (\varepsilon, \omega), Q(x):=\tilde{Q}\left(e^{x}\right) \\
K(\eta) & :=D_{\max }+\frac{\sigma g(2 b+(N+\varepsilon) R+R \eta)}{2} .
\end{aligned}
$$

All functions defined above satisfy the requirements imposed by Corollary 4.2. More specifically, using (47) and (48) we get $p_{l}^{\prime} c_{f}-p_{l}^{\prime} \mathbf{1}_{n}=-q_{l}^{\prime} v\left(\mathbf{1}_{n}\right)$ for $l=1, \ldots, N$. Since $q_{l} \in \Re_{+}^{m}$ and $v\left(\mathbf{1}_{n}\right) \in \Re_{+}^{m}$, it follows that $q_{l}^{\prime} v\left(\mathbf{1}_{n}\right) \geq 0$ and $p_{l}^{\prime} c_{f} \leq p_{l}^{\prime} \mathbf{1}_{n}$. Consequently, definition (90) implies $\eta(0)=-\varepsilon<0$. Next notice that definitions (90), (91) imply that $W(x) \geq 1, \max _{i=1, \ldots, n}\left(-x_{i}\right) \leq \ln (W(x))$ for all $x \in \Re^{n}$. Inequality (49) implies $\max _{i=1, \ldots, n}\left(e^{x_{i}}\right) \leq$ $b+N R / 2+R / 2 \sum_{l=1}^{N}\left(\max \left(p_{l}^{\prime} c_{f}-p_{l}^{\prime} e^{x}, 0\right)\right)^{2}$ for all $x \in \Re^{n}$, which combined with definition (90) gives:

$$
2 \max _{i=1, \ldots, n}\left(e^{x_{i}}\right) \leq 2 b+N R+R(\eta(x)+\varepsilon) .
$$

The above inequalities in conjunction with definition (91), allow us to conclude that the following inequalities hold

$$
\begin{aligned}
\max _{i=1, \ldots, n}\left(-x_{i}\right) & \leq \ln (W(x)), \max _{i=1, \ldots, n}\left(x_{i}\right) \\
& \leq \ln \left(b+N \frac{R}{2}+\frac{R}{2} W(x)\right)
\end{aligned}
$$

for all $x \in \Re^{n}$. Inequality (95) shows that $W \in C^{1}\left(\Re^{n} ;[1,+\infty)\right)$ as defined by (91) is radially unbounded. Using (48) and definitions (90), (91) we obtain by differentiating $\eta, W$ for all $(x, u) \in \Re^{n} \times\left[-1, D_{\max }-1\right]$ :

$$
\begin{aligned}
\sum_{i=1}^{n} \frac{\partial \eta}{\partial x_{i}}(x)\left(f_{i}(x)+g_{i}(x) u\right) & -2(1+u)(\varepsilon+\eta(x)) \\
& -2 \sum_{l=1}^{N}\left(\max \left(p_{l}^{\prime} c_{f}-p_{l}^{\prime} e^{x}, 0\right)\right) q_{l}^{\prime} v\left(e^{x}\right) \\
\sum_{i=1}^{n} \frac{\partial W}{\partial x_{i}}(x)\left(f_{i}(x)+g_{i}(x) u\right) & -(1+u)\left(2(\varepsilon+\eta(x))+\sum_{i=1}^{n} e^{-2 x_{i}}\left(c_{i, f}-e^{x_{i}}\right)\right) \\
= & 2 \sum_{l=1}^{N}\left(\max \left(p_{l}^{\prime} c_{f}-p_{l}^{\prime} e^{x}, 0\right)\right) q_{l}^{\prime} v\left(e^{x}\right) \\
& -\sum_{i=1}^{n} e^{-2 x_{i}} \sum_{j=1}^{m} S_{i, j} v_{j}\left(e^{x}\right) .
\end{aligned}
$$

Since $q_{l} \in \Re_{+}^{m}$ and $v\left(e^{x}\right) \in \Re_{+}^{m}$, it follows that $\left(\max \left(p_{l}^{\prime} c_{f}-p_{l}^{\prime} e^{x}, 0\right)\right) q_{l}^{\prime} v\left(e^{x}\right) \geq 0$ for $l=1, \ldots, N$. Therefore, we obtain from (96) and (97) for all $(x, u) \in$ $\Re^{n} \times\left[-1, D_{\max }-1\right]$ :

$$
\begin{aligned}
& \sum_{i=1}^{n} \frac{\partial \eta}{\partial x_{i}}(x)\left(f_{i}(x)+g_{i}(x) u\right) \\
& \quad \leq-2(1+u)(\varepsilon+\eta(x)) \\
& \sum_{i=1}^{n} \frac{\partial W}{\partial x_{i}}(x)\left(f_{i}(x)+g_{i}(x) u\right) \\
& \quad \leq D_{\max } \sum_{i=1}^{n} e^{-x_{i}}-\sum_{i=1}^{n} e^{-2 x_{i}} \sum_{j=1}^{m} S_{i, j} v_{j}\left(e^{x}\right) .
\end{aligned}
$$

Using (50) and (89) we get $-\sum_{i=1}^{n} e^{-2 x_{i}} \sum_{j=1}^{m} S_{i, j} v_{j}\left(e^{x}\right)$ $\leq \sum_{i=1}^{n} e^{-x_{i}} \sum_{j \in P(i)}\left|S_{i, j}\right| g\left(\max _{i=1, \ldots, n}\left(e^{x_{i}}\right)\right)$ for all $x \in \Re^{n}$. Hence, we obtain from (89), (91), (93), (94) and (99) for all $x \in \Re^{n}$ and $u \in\left[-1, D_{\max }-1\right]$ :

$$
\begin{aligned}
2 \sum_{i=1}^{n} & \frac{\partial W}{\partial x_{i}}(x)\left(f_{i}(x)+g_{i}(x) u\right) \\
& \leq\left(2 D_{\max }+\sigma g(2 b+(N+\varepsilon) R+R \eta(x))\right) \sum_{i=1}^{n} e^{-x_{i}} \\
& \leq\left(2 D_{\max }+\sigma g(2 b+(N+\varepsilon) R+R \eta(x))\right) W(x) \\
& \leq 2 K(\eta(x)) W(x) .
\end{aligned}
$$

Using (92), (98) and (100) we conclude that the function $\bar{k} \in C^{0}\left(\Re^{n} ; U\right)$ defined by (92) satisfies $\sum_{i=1}^{n} \partial W / \partial x_{i}(x)\left(f_{i}(x)+g_{i}(x) \bar{k}(x)\right) \leq K(\eta(x)) W(x)$ and $\sum_{i=1}^{n} \partial \eta / \partial x_{i}(x)\left(f_{i}(x)+g_{i}(x) \bar{k}(x)\right) \leq-\delta(\eta(x))$ for all $x \in \Re^{n}$ with $\eta(x) \geq 0$. We next notice that by virtue of the change of coordinates (87), definition (90) and the definition of $Q$ in (92), it follows that implications (24), (25), (28), and (29) are equivalent to implications Equations (54), (55), (57), and (58), respectively. Moreover, implication (56) implies implication (26). Indeed, first notice that (96) implies $\sum_{i=1}^{n} \partial \eta / \partial x_{i}(x) g_{i}(x)=-2(\varepsilon+\eta(x))<0$ for all $x \in \Re^{n}$ with $\eta(x) \geq 0$ and consequently the condition $x_{j} g_{j}(x) \sum_{l=1}^{n} \partial \eta / \partial x_{l}(x) g_{l}(x)<0$ implies $x_{j} g_{j}(x)>0$, or equivalently, $\min \left(c_{i, f}, 1\right)<c_{i}<\max \left(c_{i, f}, 1\right)$. Implication (26) requires $\sum_{l=1}^{n} \partial \eta / \partial x_{l}(x)\left(f_{l}(x)+g_{l}(x) u\right) \leq-\delta(\eta(x))$

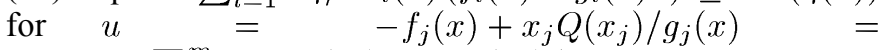
$-1-e^{-x_{j}} \sum_{l=1}^{m} S_{j, l} v_{l}\left(e^{x}\right)+x_{j} Q\left(x_{j}\right) / c_{j, f} e^{-x_{j}}-1$.

Notice that (56) implies that $\omega / 2 \varepsilon$ $-c_{j} \ln \left(c_{j}\right) \tilde{Q}\left(c_{j}\right)+\sum_{l=1}^{m} S_{j, l} v_{l}(c) / c_{j, f}-c_{j}-\leq$ inequality (98) combined with definition (92) gives $\sum_{i=1}^{n} \partial \eta / \partial x_{i}(x)\left(f_{i}(x)+g_{i}(x) u\right) \leq-\omega \leq-\delta(\eta(x))$ for all $x \in \Re^{n}$ with $0 \leq \eta(x) \leq \varepsilon$. Consequently, implication (26) holds.

We next show that implication (27) holds. By virtue of (100) it suffices to show that for all $x \in \Re^{n}$ with $\max _{s=1, \ldots, n} \tilde{\gamma}_{j, s}\left(\left|x_{s}\right|\right) \leq$ $\left|x_{j}\right|, 0 \leq \eta(x) \leq \varepsilon$ :

- $-f_{j}(x)+x_{j} Q\left(x_{j}\right) / g_{j}(x) \leq D_{\max }-1$ for $x_{j} g_{j}(x)<0$ and $\sum_{l=1}^{n} \partial W / \partial x_{l}(x) g_{l}(x)>0$,

- $-f_{j}(x)+x_{j} Q\left(x_{j}\right) / g_{j}(x) \geq-1$ for $x_{j} g_{j}(x)>0$ and $\sum_{l=1}^{n} \partial W / \partial x_{l}(x) g_{l}(x)<0$ 
The above conditions are implied by the following conditions for all $c \in \operatorname{int}\left(\Re_{+}^{n}\right)$ with $\max _{s=1, \ldots, n} \tilde{\gamma}_{i, s}\left(\left|\ln \left(c_{s}\right)\right|\right) \leq\left|\ln \left(c_{i}\right)\right|$, $\sum_{l=1}^{N}\left(\max \left(p_{l}^{\prime} c_{f}-p_{l}^{\prime} c, 0\right)\right)^{2} \leq 2 \varepsilon$ :

- $-\sum_{l=1}^{m} S_{j, l} v_{l}(c)+c_{j} \tilde{Q}\left(c_{j}\right) \ln \left(c_{j}\right) / c_{j, f}-c_{j} \leq D_{\max }$ for $c_{j}>\max \left(c_{j, f}, 1\right)$ or $\min \left(c_{j, f}, 1\right)>c_{j}$,

- $0 \leq-\sum_{l=1}^{m} S_{j, l} v_{l}(c)+c_{j} \tilde{Q}\left(c_{j}\right) \ln \left(c_{j}\right) / c_{j, f}-c_{j}$ for $\min \left(c_{j, f}, 1\right)<c_{j}<\max \left(c_{j, f}, 1\right)$

The above conditions are direct consequences of (57) and (58). Thus we conclude that implication (27) holds.

\section{REFERENCES}

[1] D. Angeli, "A tutorial on chemical reaction network dynamics," Euro. J. Control, vol. 15, no. 3-4, pp. 398-406, 2009.

[2] Z. Artstein, "Stabilization with relaxed controls," Nonlinear Analysis: Theory, Methods and Applicat, vol. 7, pp. 1163-1173, 1983.

[3] R. Bellman, "Vector Lyapunov functions," SIAM J. Control, vol. 1, pp. 32-34, 1962.

[4] G. Craciun and M. Feinberg, "Multiple equilibria in complex chemical reaction networks: I. the injectivity property," SIAM J. Appl. Mathematics, vol. 65, no. 5, pp. 1526-1546, 2006.

[5] Dashkovskiy, S. B. Rüffer, and F. Wirth, "Small gain theorems for large scale systems and construction of ISS Lyapunov functions," SIAM J. Control Optim., vol. 48, no. 6, pp. 4089-4118, 2010.

[6] R. A. Freeman and P. V. Kokotovic, Robust Nonlinear Control Design-State Space and Lyapunov Techniques. Boston, USA: Birkhauser, 1996.

[7] W. M. Haddad, V. Chellaboina, and S. G. Nersesov, "Vector dissipativity theory and stability of feedback interconnections for largescale non-linear dynamical systems," Int. J. Control, vol. 77, no. 10, pp. 907-919, 2004.

[8] W. M. Haddad and V. Chellaboina, Nonlinear Dynamical Systems and Control. A Lyapunov-Based Approach. Princeton, NJ: Princeton Univ. Press, 2008.

[9] Y. G. Hong and D. Cheng, Analysis and Control of Nonlinear Systems. Beijing, China:, 2005.

[10] H. Ito, Z. P. Jiang, S. Dashkovskiy, and B. S. Rüffer, "Robust stability of networks of iISS systems: Construction of sum-type Lyapunov functions," IEEE Trans. Autom. Control, vol. 58, no. 5, pp. 1192-1207, May 2013,

[11] Z. P. Jiang, I. M. Y. Mareels, and Y. Wang, "A Lyapunov formulation of the nonlinear small-gain theorem for interconnected systems," $\mathrm{Au}$ tomatica, vol. 32, pp. 1211-1214, 1996.

[12] Z.-P. Jiang, "Decentralized control for large-scale nonlinear systems: A review of recent results," J. Dynam. Contin., Discrete Impulsive Syst., vol. 11 , pp. 537-552, 2004

[13] I. Karafyllis, C. Kravaris, L. Syrou, and G. Lyberatos, "A vector Lyapunov function characterization of input-to-State stability with application to robust global stabilization of the chemostat," Eur. J. Control, vol. 14 , no. 1 , pp. 47-61, 2008.

[14] I. Karafyllis and Z.-P. Jiang, "A vector small-gain theorem for general nonlinear control systems," IMA J. Math. Control Inf., vol. 28, no. 3 , pp. 309-344, 2011.

[15] I. Karafyllis and Z.-P. Jiang, "A new small-gain theorem with an application to the stabilization of the chemostat," Int. J. Robust Nonlinear Control, vol. 22, no. 14, pp. 1602-1630, 2012.

[16] I. Karafyllis and Z.-P. Jiang, Stability and Stabilization of Nonlinear Systems, ser. Communications and Control Engineering. London, U.K.: Springer-Verlag, 2011.

[17] M. Krstic, I. Kanellakopoulos, and P. V. Kokotovic, Nonlinear and Adaptive Control Design. Hobokon, NJ: Wiley, 1995.

[18] V. Lakshmikantham and X. Liu, Stability Analysis in Terms of Two Measures. Singapore: World Scientific, 1993.

[19] V. Lakshmikantham, V. M. Matrosov, and S. Sivasundram, Vector Lyapunov Functions and Stability Analysis of Nonlinear Systems. Dordrecht, The Netherlands: Kluwer Academic, 1991.

[20] Y. Lin, E. D. Sontag, and Y. Wang, "A smooth converse Lyapunov theorem for robust stability," SIAM J. Control Optim., vol. 34, pp. 124-160, 1996.

[21] T. Liu, D. J. Hill, and Z. P. Jiang, "Lyapunov formulation of ISS smallgain in continuous-time dynamical networks," Automatica, vol. 47, pp. 2088-2093, 2011.
[22] M. Malisoff and F. Mazenc, Constructions of Strict Lyapunov Functions. London, U.K.: Springer-Verlag, 2009.

[23] A. N. Michel, "On the status of stability of interconnected systems," IEEE Trans. Circuits Syst., vol. CS-30, no. 6, pp. 326-340, 1983.

[24] S. G. Nersesov and W. M. Haddad, "On the stability and control of nonlinear dynamical systems via vector Lyapunov functions," IEEE Trans. Autom. Control, vol. 51, no. 2, pp. 203-215, Feb. 2006.

[25] S. G. Nersesov, W. M. Haddad, and Q. Hui, "Finite-time stabilization of nonlinear dynamical systems via control vector Lyapunov functions," J. Franklin Inst., vol. 345, pp. 819-837, 2008.

[26] S. G. Nersesov and W. M. Haddad, "Control vector Lyapunov functions for large-scale impulsive dynamical systems," Nonlinear Analysis: Hybrid Syst, vol. 1, pp. 223-243, 2007.

[27] D. Siljak, Decentralized Control of Complex Systems. New York: Academic, 1991.

[28] H. Smith and P. Waltman, The Theory of the Chemostat. Dynamics of Microbial Competition, Cambridge Studies in Mathematical Biology, 13. Cambridge, U.K.: Cambridge Univ. Press, 1995.

[29] E. D. Sontag, "Universal" construction of Artstein's theorem on nonlinear stabilization," Syst. Control Lett., vol. 13, pp. 117-123, 1989.

[30] J. Tsinias, "Sufficient Lyapunov-like conditions for stabilization," Math. Control, Signals Syst., vol. 2, pp. 343-357, 1989.

[31] A. Van der Schaft, S. Rao, and B. Jayawardhana, "On the Mathematical Structure of Balanced Chemical Reaction Networks Governed by Mass Action Kinetics," arXiv:1110.6078v1 [math.OC].

[32] V. I. Vorotnikov, Partial Stability and Control. Boston, MA Birkhauser, 1998.

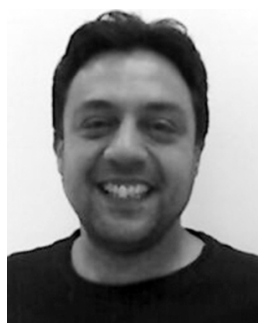

Iasson Karafyllis received the B.S. degree in chemical engineering from the National Technical University of Athens (NTUA), Athens, Greece, in 1994, the M.Sc. degree in mathematics from the University of Minnesota, Minneapolis, MN, USA, in 1997, and the Ph.D. degree in mathematics from the NTUA, in 2003.

$\mathrm{He}$ is currently an Assistant Professor in the Department of Mathematics at NTUA. His research interests include mathematical systems and contro theory, stability theory, and robust feedback stabilization problems for deterministic systems. He is a coauthor of the book Stability and Stabilization of Nonlinear Systems, (with Zhong-Ping Jiang, Springer, 2011).

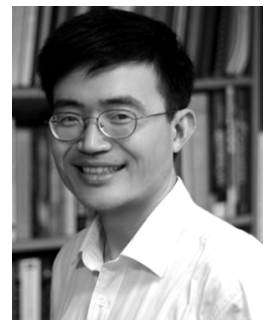

Zhong-Ping Jiang (M'94-SM'02-F'08) received the B.Sc. degree in mathematics from the University of Wuhan, Wuhan, China, in 1988, the M.Sc. degree in statistics from the University of Paris XI, Paris, France, in 1989, and the Ph.D. degree in automatic control and mathematics from the Ecole des Mines de Paris, France, in 1993.

Currently, he is a Full Professor of electrical and computer engineering at the Polytechnic Institute of New York University, Brooklyn, NY, USA. His main research interests include stability theory, robust, adaptive and distributed nonlinear control, adaptive dynamic programming and their applications to information, mechanical, and biological systems. He is coauthor of the book Stability and Stabilization of Nonlinear Systems (with Dr. Iasson Karafyllis, Springer 2011). He is an Editor for the International Journal of Robust and Nonlinear Control and has served as an Associate Editor for several journals including Mathematics of Control, Signals and Systems, Systems and Control Letters, IEEE TRANSACTIONS ON AUTOMATIC CONTROL, and the European Journal of Control and Science China: Information Sciences.

Dr. Jiang is a recipient of the prestigious Queen Elizabeth II Fellowship Award from the Australian Research Council, the CAREER Award from the U.S. National Science Foundation, and the Young Investigator Award from the National Natural Science Foundation of China. Recent awards recognizing his research work include the Best Theory Paper Award (with Y. Wang) at the 2008 WCICA, and the Guan Zhao Zhi Best Paper Award (with T. Liu and D. Hill) at the $2011 \mathrm{CCC}$, and the Shimemura Young Author Prize (with his student Yu Jiang) at the 2013 Asian Control Conference in Istanbul, Turkey. Prof. Jiang is a Fellow of IFAC. 